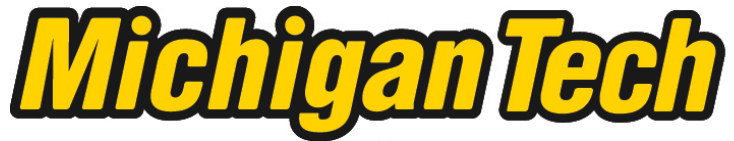 \\ Michigan Technological University Create the Future Digital Commons @ Michigan Tech
}

Dissertations, Master's Theses and Master's Reports - Open

Dissertations, Master's Theses and Master's

Reports

2013

\section{Molecular Modeling of PMR-15 Polyimide}

FNU Pruthul Kokkada Ravindranath

Michigan Technological University

Follow this and additional works at: https://digitalcommons.mtu.edu/etds

Part of the Materials Science and Engineering Commons, and the Mechanical Engineering Commons Copyright 2013 FNU Pruthul Kokkada Ravindranath

\section{Recommended Citation}

Pruthul Kokkada Ravindranath, FNU, "Molecular Modeling of PMR-15 Polyimide", Master's Thesis, Michigan Technological University, 2013.

https://doi.org/10.37099/mtu.dc.etds/598

Follow this and additional works at: https://digitalcommons.mtu.edu/etds

Part of the Materials Science and Engineering Commons, and the Mechanical Engineering Commons 
By

Pruthul Kokkada Ravindranath

\begin{abstract}
A THESIS
Submitted in partial fulfillment of the requirements for the degree of MASTER OF SCIENCE

In Mechanical Engineering
\end{abstract}

MICHIGAN TECHNOLOGICAL UNIVERISITY

2013

(C) 2013 Pruthul Kokkada Ravindranath 
This thesis has been approved in partial fulfillment of the requirement for the Degree of MASTER OF SCIENCE in Mechanical Engineering.

Department of Mechanical Engineering - Engineering Mechanics

Thesis Advisor: Dr. Gregory Odegard

Committee Member: Dr. Ibrahim Miskioglu

Committee Member: Dr. Ranjit Pati

Department Chair: Dr. William Predebon 


\section{TABLE OF CONTENTS}

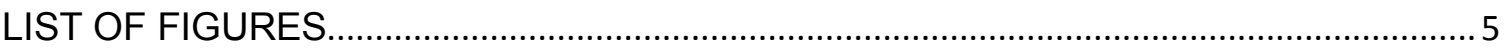

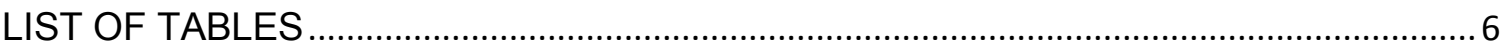

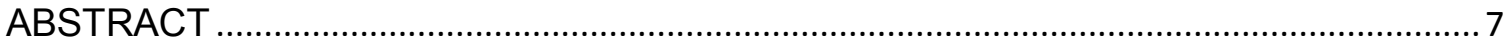

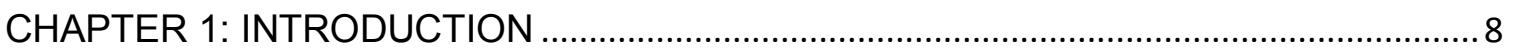

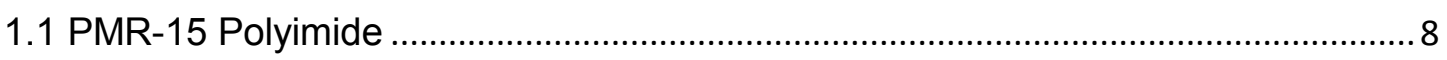

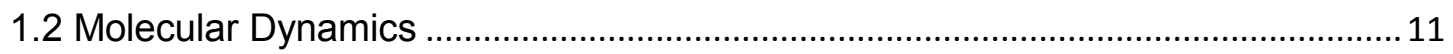

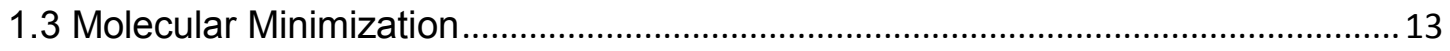

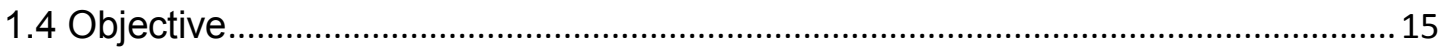

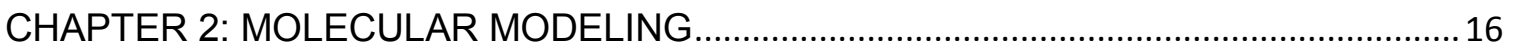

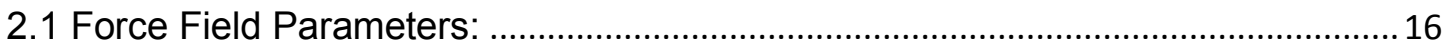

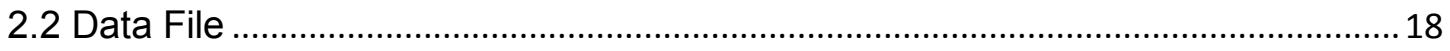

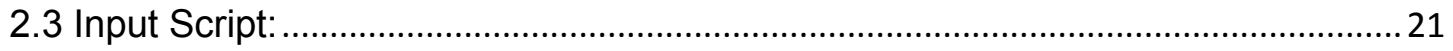

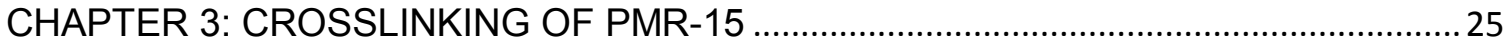

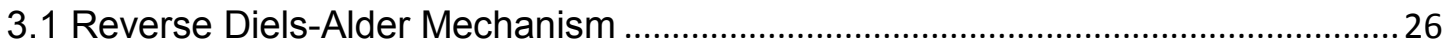

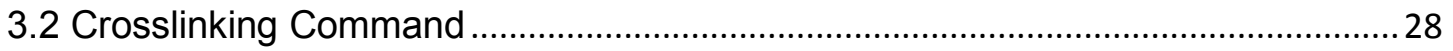

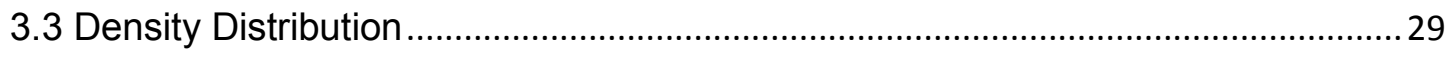

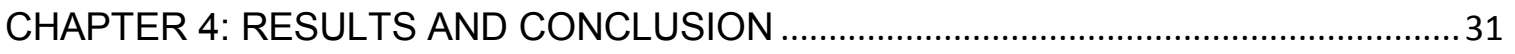

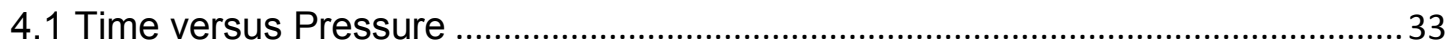

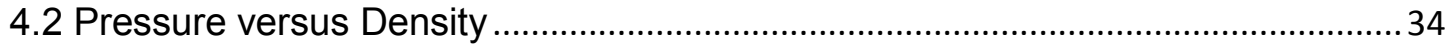

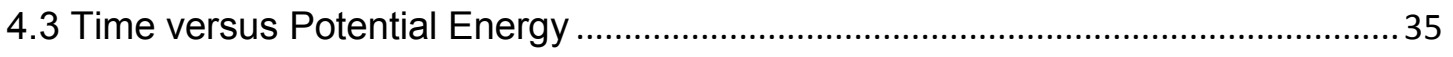

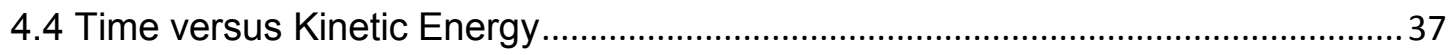

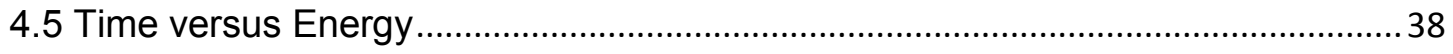

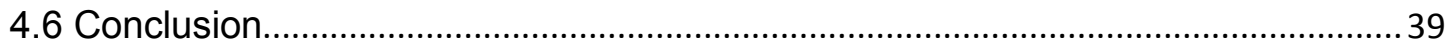




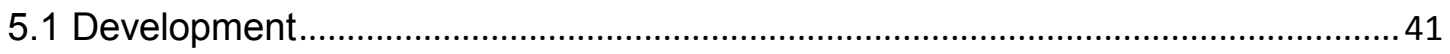

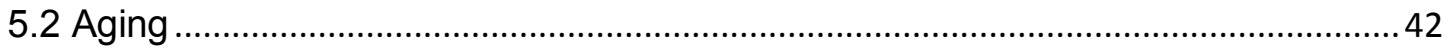

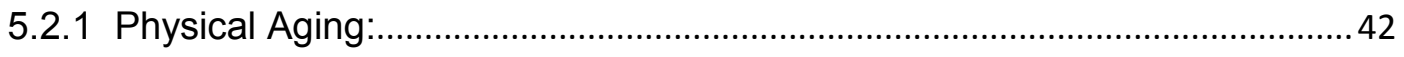

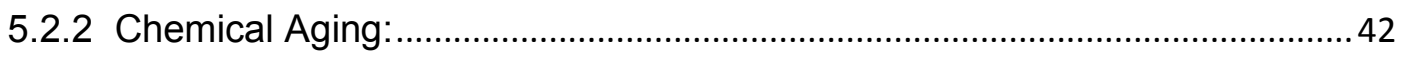

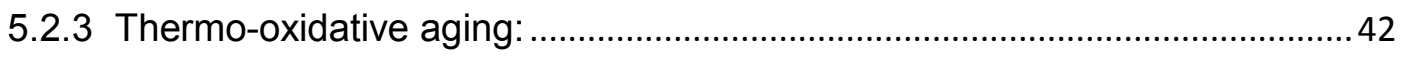

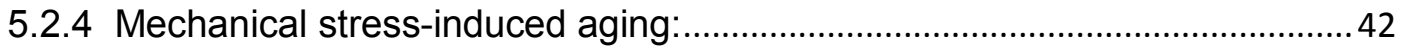

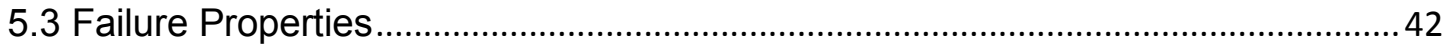

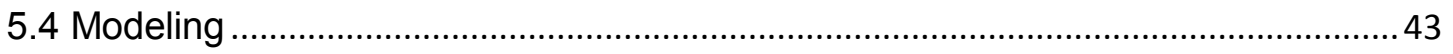

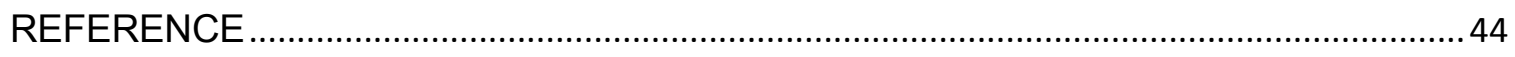

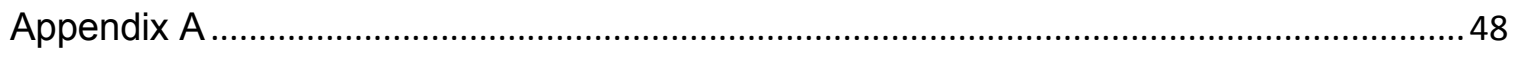

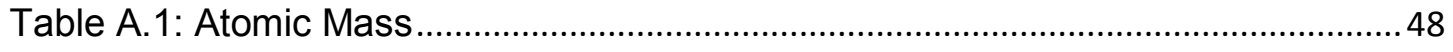

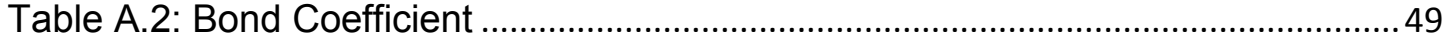

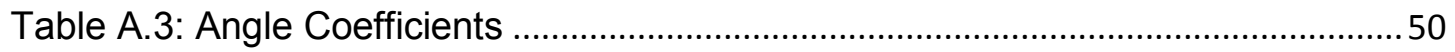

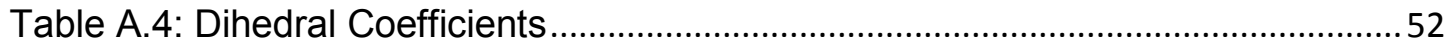

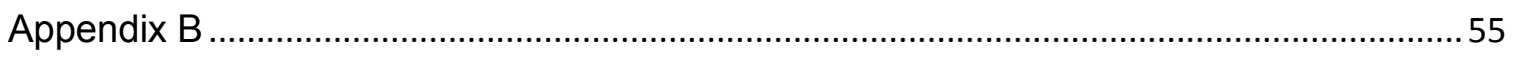

B.1 Molecular Energy Minimization and Deform Simulation ........................................5 55

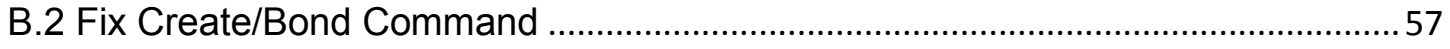

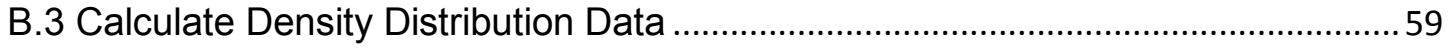

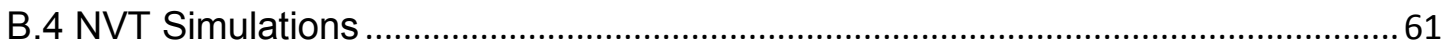

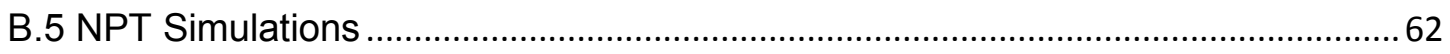

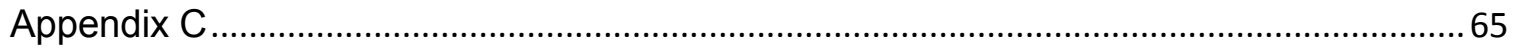




\section{LIST OF FIGURES}

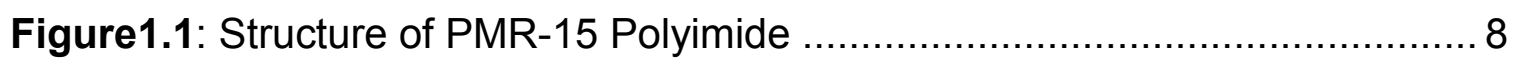

Figure 1.2: Formation of polyimide PMR-15 from oligomers ........................... 10

Figure 1.3: Algorithm of a computational energy minimization procedure ........ 14

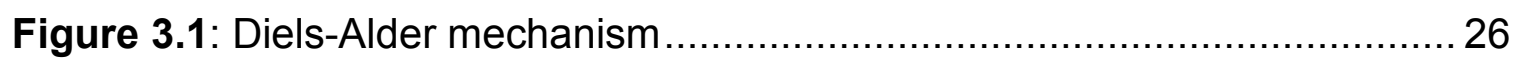

Figure 3.2: Reverse Diels-Alder mechanism ........................................... 26

Figure 3.3: Reverse Diels-Alder Reaction of the Noroborene end-caps ........... 27

Figure 3.4: Density distribution chart along $X, Y$ and $Z$ Axes ..........................2 29

Figure 4.1: Structure of a single PMR-15 chain ............................................. 32

Figure 4.2: PMR-15 model of 150 chains in a simulation box.......................... 32

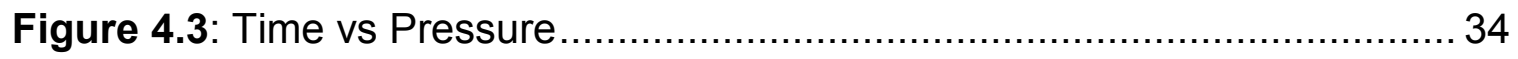

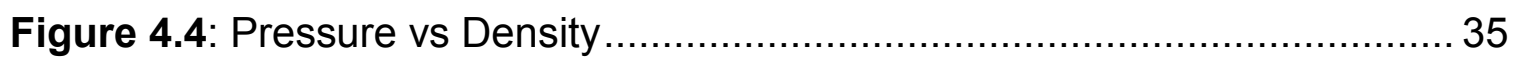

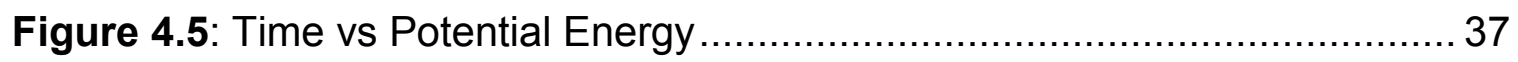

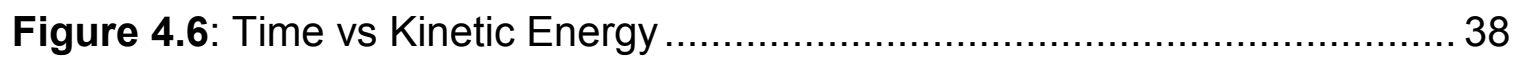

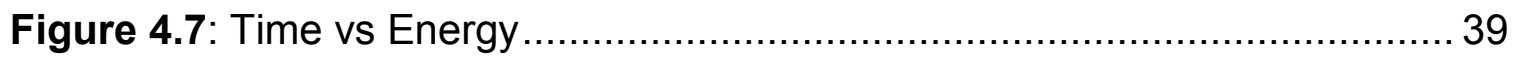




\section{LIST OF TABLES}

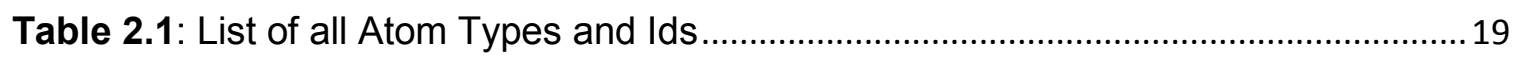




\section{ABSTRACT}

PMR-15 polyimide is a polymer that is used as a matrix in composites. These composites with PMR-15 matrices are called advanced polymer matrix composite that is abundantly used in the aerospace and electronics industries because of its high temperature resistivity. Apart from having high temperature sustainability, PMR-15 composites also display good thermal-oxidative stability, mechanical properties, processability and low costs, which makes it a suitable material for manufacturing aircraft structures. PMR-15 uses the reverse Diels-Alder (RDA) method for crosslinking which provides it with the groundwork for its distinctive thermal stability and a range of $280-300^{\circ} \mathrm{C}$ use temperature. Regardless of such desirable properties, this material has a number of limitations that compromises its application on a large scale basis. PMR-15 composites has been known to be very vulnerable to micro-cracking at inter and intra-laminar cracking. But the major factor that hinders its demand is PMR-15's carcinogenic constituent, methylene dianilineme (MDA), also a liver toxin. The necessity of providing a safe working environment during its production adds up to the cost of this material. In this study, Molecular Dynamics and Energy Minimization techniques are utilized to simulate a structure of PMR-15 at a given density of $1.324 \mathrm{~g} / \mathrm{cc}$ and an attempt to recreate the polyimide to reduce the number of experimental testing and hence subdue the health hazards as well as the cost involved in its production. Even though this study does not involve in validating any mechanical properties of the model, it could be used in future for the validation of its properties and further testing for different properties like aging, microcracking, creep etc. 


\section{CHAPTER 1: INTRODUCTION}

\subsection{PMR-15 Polyimide}

Advanced Polymer Matrix composites are high performance materials that are extensively used for their excellent mechanical and physical properties like high stiffness, light weight, high modulus of elasticity, high strength-to-weight ratio, etc. However, low thermal stability of about $130^{\circ} \mathrm{C}$, sourced the necessity to overcome this problem (Hay, Boyle et al. 1989).

During 1960's, NASA's Lewis Research Center developed a type of advanced polymer matrix composite, called condensed polyimides. Condensed polyimides retained the same mechanical and physical properties as its predecessors but also had the advantage over them of high thermo-oxidative stability and a resistance to temperatures up to $316^{\circ} \mathrm{C}$ when exposed for thousands of hours. Presently, PMR-15 composite is the leading condensed polyimide, developed by NASA, extensively used in aerospace and aviation industries (Figure 1.1).

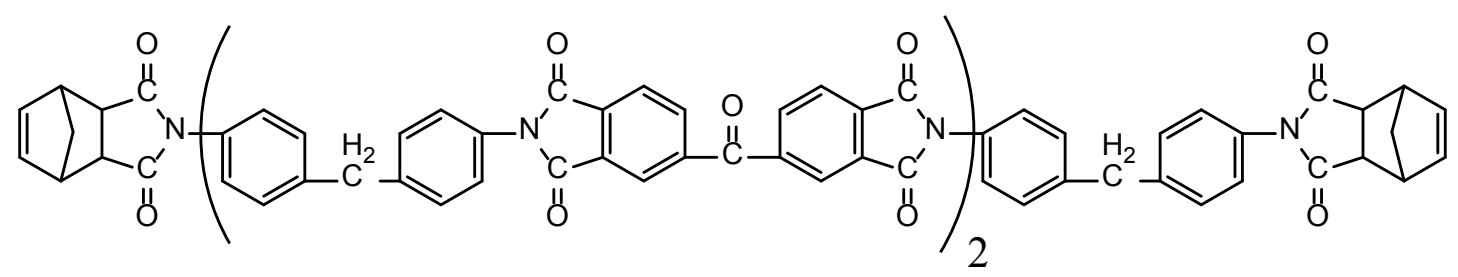

Figure1.1: Structure of PMR-15 Polyimide

Due to the complications in the processing of polymers having heterocyclic and aromatic groups, Polymerization of Monomeric Reactants (PMR) approach was developed (Serafini, Delvigs et al. 1972). The PMR approach for processing PMR-15 comprises of dimethyl ester of 3,3',4,4'-benzophenone tetracarboxylic acid (BTDE), methylene dianiline (MDA), and monoalkylester of 5-noroborene2,3-dicarboxylic acid (NE) in a molar ratio of 2.087:3.087:2 respectively (Dangarwala 1993). This molar ratio adds up to a molecular weight of 1500 which 
gives the polymer its name, PMR-15, and also a good thermo-oxidative stability, mechanical and physical properties. In this process, developed by NASA Lewis Research Center, an aromatic diamine, a diester of tetra-carboxylic acid and a monoalkylester of a dicarboxylic acid are made to dissolve in a polar solvent, usually methanol. This mixture is impregnated to reinforced plastic fibers and fabric and a prepreg is formed by removing excess methanol. Polymerization of monomers occur at temperatures around $150-200^{\circ} \mathrm{C}$ and further crosslinking takes place at around $280-320^{\circ} \mathrm{C}$ by reverse Diels-Alder (RDA) method. Thermal imidization begins as soon as the monomers are mixed together at the required ratio. When heated, formation of polyamic acid takes place and methanol is released. On further heating, imidization takes place to form the imide. These imides crosslink to each other when pressure and still more temperature is introduced to the system and forms a polyimide. Because of the insufficient data on the curing process, various industries use different kinds of curing cycles.

To create PMR-15 composites, it is necessary to first produce fine preimpregnated fiber-reinforced plastics (prepregs). The fibers in these prepregs are placed in between the layers of release film. Then by using vacuum bag autoclave process or simply by using compression, the prepregs are cast into laminates. Impurities like tri-esters, acids, undesirable isomers etc. can affect the material properties. High importance should be given to the processing methods like polymerization and rheology to manufacture defect less laminates. Understanding the resin viscosity, which depends on time, temperature, prepeg formulations etc. and its variance during the curing process could help in producing better laminates. The polymerization of PMR-15 takes place in three steps: formation of polyamic acid, imidization, and crosslinking (Dangarwala 1993). The chemical reaction taking place in the formation of PMR-15 is shown in Figure 1.2. 
<smiles>CC(=O)c1ccc(C(=O)c2ccc(C(=O)O)c(C(=O)O)c2)cc1C(=O)O</smiles>

NE

MDA

BTDE

$-\mathrm{H}_{2} \mathrm{O},-\mathrm{CH}_{3} \mathrm{OH}$<smiles>CCN1C(=O)C2C3C=CC(C3)C2C1=O</smiles>

Heat, Pressure

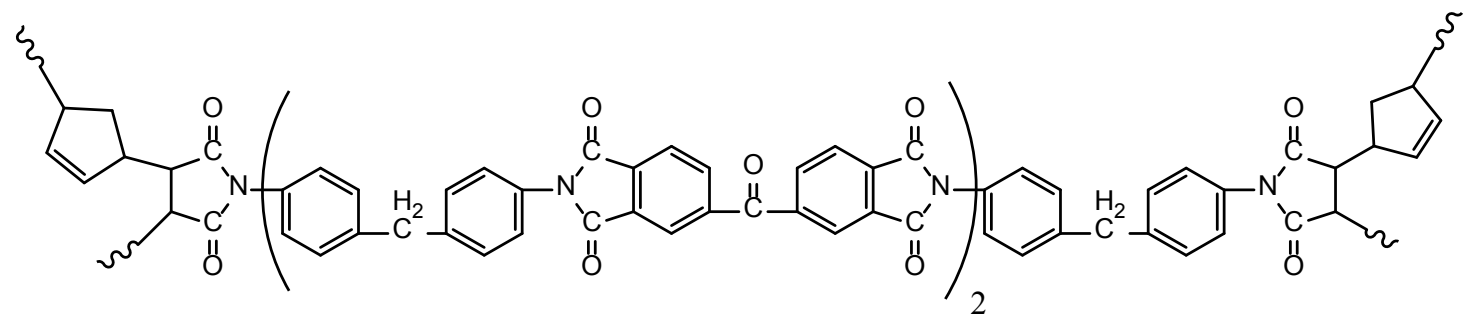

Figure 1.2: 3 -step reaction for formation of polyimide PMR-15 from oligomers.

The chemical structure of PMR-15 constitutes the thermal and mechanical properties of PMR-15. The heterocyclic and aromatic rings present in the structure contribute to the thermal stability and the high stiffness ratio of the polyimide, while the thermo-oxidative stability of the polyimide is governed by the crosslinking type and density of the material. 
The crosslinking mechanism of PMR-15 takes place by the RDA type of reaction. Lower rates of RDA reaction can be achieved at lower temperatures which leads to a consistency in the physical properties. However for systems with higher cure temperature, due to the methylene radical production, the rate of crosslinking increases and so does the density of the system which results in a system of high modulus.

It is very important for any material to have their mechanical and physical properties validated at room as well as augmented temperatures. Wide research has been done on the mechanical behavior of unidirectional composites with PMR-15 matrix, like those of effects of aging at high temperatures, thermo-oxidative stability, elastic and plastic deformations. These tests have resulted in confirming significant changes in the elastic, plastic and tensile strength properties of the composites at very high temperatures (Odegard 2000). It has also been observed that under loads perpendicular to the direction of the fibers and in shear, unidirectional polymer-matrix composite materials tend to show non-linear mechanical behavior. This non-linear behavior of these materials becomes more prominent as the temperature moves more towards the glass transition temperature of the material.

\subsection{Molecular Dynamics}

Molecular Dynamics can be defined as the computer simulation technique used to study the motion of atoms by allowing the atoms and molecules to interact over a small period of time by following the classic laws of physics. Molecular dynamics makes use of simulations of atoms and molecules to predict the properties of the system at the macroscopic level. This relationship between position and velocities of the atoms and the macroscopic properties is determined by statistical mechanics which makes use of accurate mathematical formulas. MD simulations make use of these mathematical formulas to solve the equations 
of motion of the atoms to also determine the thermodynamic as well as the time dependent properties.

An ensemble is defined by the Oxford dictionary as "a group of similar systems, or different states of the same system often considered statistically". In case of MD simulations, these systems with different microscopic states will be considered to have the same macroscopic or thermodynamic state. Generally there are four different types of ensembles in MD simulations:

1. Microcanonical ensemble (NVE): This is an adiabatic type of thermodynamic state, where the number of atoms (N), volume (V) and energy $(E)$ are fixed and hence no heat exchange takes place. It relates to an isolated system where the total energy is conserved.

2. Canonical ensemble (NVT): In this type of ensemble the number of atoms (N) and volume $(V)$ are kept constant while the temperature $(T)$ is defined by the same temperature that the system would have in equilibrium. This type of ensemble differs from the microcanonical ensemble in the aspect of energy. While in the former type of ensemble, the energy of each system is fixed, this type has its average energy fixed.

3. Isothermal-Isobaric ensemble (NPT): As the name suggests, this is the type of ensemble where both the temperature $(T)$ and the pressure $(P)$ of the system with fixed number of atoms $(\mathrm{N})$ are kept constant using a thermostat and a barostat. To achieve this constant temperature and pressure of the system within a given window, the change in volume of the system is allowed.

4. Isoenthalpic-Isobaric ensemble (NPH): In this ensemble, for a fixed number of atoms $(\mathrm{N})$, the enthalpy $(\mathrm{H})$ and the pressure $(\mathrm{P})$ of the system are kept constant. Using the formula for enthalpy of a homogenous system:

$$
\mathrm{H}=\mathrm{U}+\mathrm{pV}
$$

where, $\mathrm{H}$ = enthalpy; $\mathrm{U}=$ internal energy; $\mathrm{p}=$ pressure; $\mathrm{V}=$ volume the enthalpy is kept constant by mainly varying the volume and the internal energy of system. 


\subsection{Molecular Minimization}

Along with the above ensembles in MD simulations, Energy Minimization methods were also used to achieve the equilibrium state of the molecules. For a system in non-equilibrium state, energy minimization process, engages highly complicated mathematical equations and functions to relocate the atoms with the purpose of optimizing the energies in a system to a minimum level. These functions are used along with some basic algorithms to continuously check for the net forces acting on different particles, re-assigning them into trial locations, and again continuing the procedure until a minimum energy level is achieved for the whole system. The algorithm in Figure 2.1 gives a schematic procedure of how energy minimization functions: 


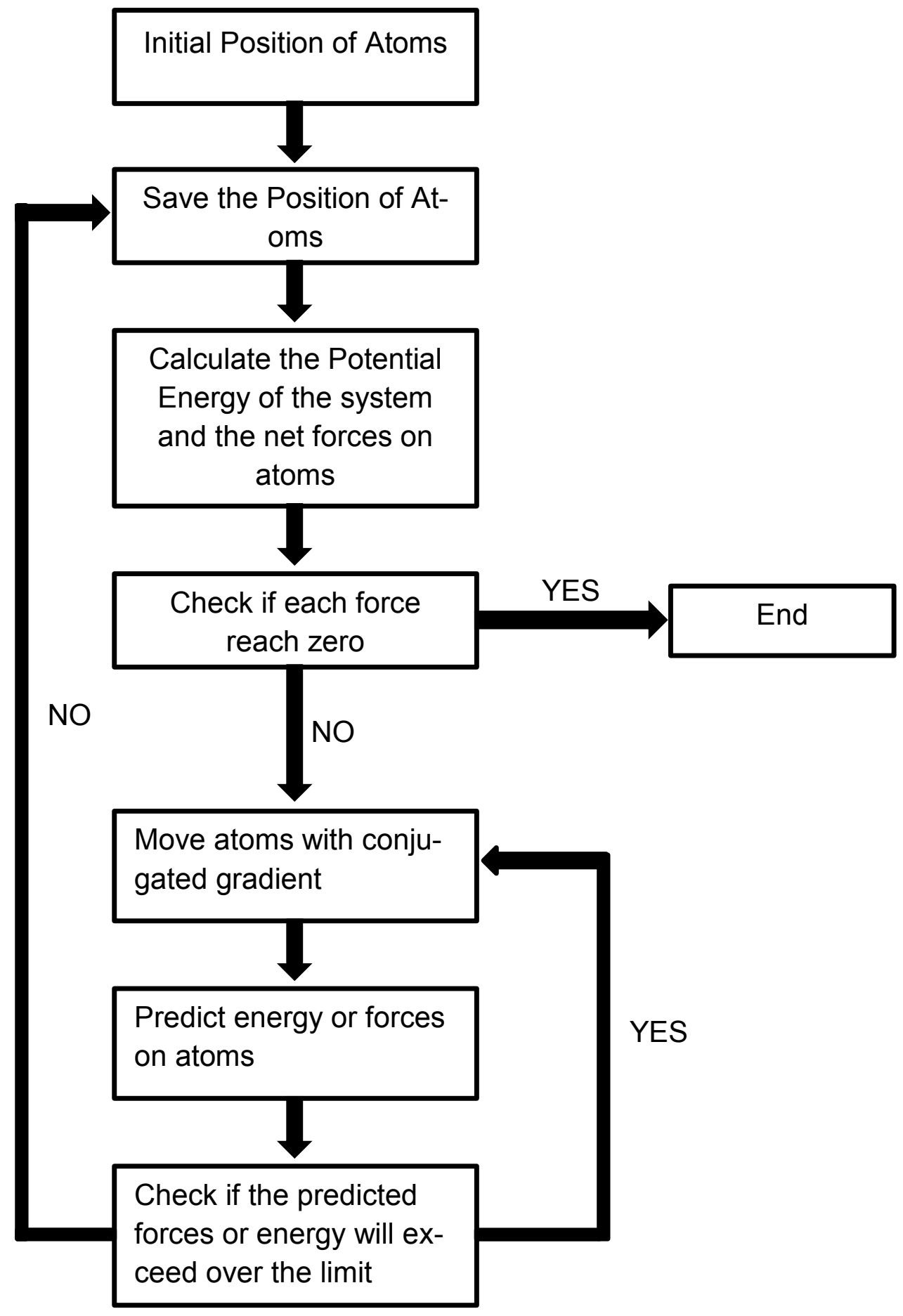

Figure 1.3: Algorithm of a computational energy minimization procedure (See Appendix C) 


\subsection{Objective}

The objective of this research is to create a molecular model of PMR-15 with a density of $1.324 \mathrm{~g} / \mathrm{cc}$ (Scola, D. A., Ployimide Resins, University of Connecticut, 105-119) using the OPLS-All Atoms force field parameters. One of the most important factors of this research is to validate if the use of OPLS-All Atoms force field parameters in modeling PMR-15, provides us with a molecular model that remains stable under different conditions or not. A careful and detailed approach was used to model this system of 25350 atoms and with high crosslinking ratio of up to $95 \%$. Chapter 2 and 3 discusses about the molecular modeling procedures adapted in this research, the crosslinking process of PMR-15, different commands used and the density distribution after crosslinking of the final system. Chapter 4 discusses the results of the simulations and how the system was validated by introducing the system to temperatures and pressure of ambient conditions. 


\section{CHAPTER 2: MOLECULAR MODELING}

Molecular Modeling using LAMMPS has to follow a specific set of steps. These steps usually comprises of the following:

- Recognizing the force field parameters

- Creating Data file

- Writing an Input script for LAMMPS to follow

- Running the Simulation

All of these steps concentrate around the input script, where the commands are mentioned for LAMMPS to follow. But before the commands are written to create the input script, it is important to create a data file which is read by LAMMPS to receive the geometric properties and spatial information of the atoms and molecules. These information on the properties of the atoms was collected from the OPLS-All Atoms force field parameters provided by TINKER molecular modeling software (Poder, J. W., et al., 1987). This data was then utilized to create the data file which was read by the input script and executed by LAMMPS.

\subsection{Force Field Parameters:}

In correspondence to molecular modeling, force field parameters are a set of mathematical formulas and constants used to define the potential energy of a system of molecules and atoms. Extensive experiments and high level quantum mechanical calculations are performed to derive the force field parameters (Ponder JW et al., 2003). In the past, the use of OPLS force field parameters have been proven to be effective in modeling as well as performing various other tests on the created molecular models of epoxies (Bandyopadhyay, A., 2012). So, to check if the same is applicable for polyimides, OPLS force field parameters are used in this modeling.

Jorgensen et al. developed the Optimized Potentials for Liquid Simulations (OPLS) force fields at Purdue University and later at Yale University. There are two types of OPLS force field parameters (Jorgensen and Tirado-Rives 1988): 
1. OPLS United Atoms (UA)

2. OPLS All Atoms (AA)

OPLS-UA force field is that parameter which considers Hydrogen atoms next to Carbon atoms within the Carbon atom parameters completely. This reduces the simulation time.

OPLS-AA force field, on the other hand includes the parameters of every atom in the system explicitly.

OPLS-AA force field parameter is used here to model PMR-15, to check if the created molecular model can be validated under ambient conditions or not.

In this case, the functional form for the total potential energy is given by the equation:

$$
E(\phi)=E_{\text {bond }}(\phi)+E_{\text {angle }}(\phi)+E_{\text {non-bonded }}(\phi)+E_{\text {torsion }}(\phi)
$$

- $E_{\text {bond }}$ is the Bond Energy and is given by:

$$
E_{\text {bond }}=\sum_{\text {bonds }} K_{r}\left(r-r_{e q}\right)^{2}
$$

Where, $K_{r}=$ force constant, $\mathrm{r}=$ distance between the two bonded atoms, and $r_{e q}=$ equilibrium bond distance (Refer Appendix A.2).

- $E_{\text {angle }}$ is the Angle Energy, i.e. the energy in relation with bond-angle bending and is given by:

$$
E_{\text {angle }}=\sum_{\text {angles }} K_{\theta}\left(\theta-\theta_{e q}\right)^{2}
$$

Where, $K_{\theta}=$ force constant, $\theta=$ bond angle between two bonds, and $\theta_{e q}=$ equilibrium bond angle (Refer Appendix A.3).

- $E_{n o n-b o n d e d}$ is the Non-Bonded Energy and consists of the dielectric energies and the energies due to the van der Waal's interactions. Because the dielectric 
values of the atoms were assumed not to change during the simulation, due to their small values, the dielectric energy for the system was found to be zero. So, only the van de Waal's energies were considered in this process. The equation for van der Waal's energy is given by:

$$
E_{v a n-d e r-W a a l s}=\sum_{n o n \text { bonded paris }}\left(\frac{A_{i k}}{r_{i k}^{12}}-\frac{C_{i k}}{r_{i k}^{6}}\right)
$$

Where, $\mathrm{A}$ and $\mathrm{C}$ are the atom type dependent constants and $\mathrm{r}$ is the distance between them.

- $E_{\text {torsion }}$ is the Torsional or dihedral Energy and is given by:

$$
\begin{array}{r}
E_{\text {torsion }}=\sum_{i}\left\{\frac{V_{1}^{i}}{2}\left[1+\cos \left(\phi_{i}\right)\right)\right]+\frac{V_{2}^{i}}{2}\left[1+\cos \left(2 \phi_{i}\right)\right]+ \\
\left.\frac{V_{3}^{i}}{2}\left[1+\cos \left(3 \phi_{i}\right)\right]+\frac{V_{4}^{i}}{2}\left[1+\cos \left(4 \phi_{i}\right)\right]\right\}
\end{array}
$$

Where, $\varphi_{i}=$ dihedral angle, $V_{1}, V_{2}, V_{3} \& V_{4}=$ coefficients in the Fourier series (Refer Appendix A.4). A dihedral is formed between four consecutive atoms in a chain. The angle between the planes made by the first three atoms in the group of four atoms, and the last three atoms is called a dihedral angle. The sum of this series of each dihedral angle gives the total torsional energy for the system (Jorgensen, Maxwell et al. 1996).

\subsection{Data File}

The structure of a single un-crosslinked PMR-15 monomer was modeled in reference to the structure provided by (Hay, Boyle et al. 1989), as shown in Figure 1.1. 169 atoms were identified in a single monomer of PMR-15. Using the OPLS- 
AA force field parameters provided by TINKER molecular modeling software (Poder, J. W., et al., 1987 ), each atom in the PMR-15 were grouped in separate atom type categories, depending on various factors like hybridization, neighboring atom(s), molecular groups, number of bonds, etc. Thirteen different atom types were categorized. Two extra atom types were included in the list based on the fact of them, being the crosslinking sites. Table 2.1, below lists down each 169 atoms and their corresponding atom types:

Table 2.1: List of all Atom Types and Ids

\begin{tabular}{|c|c|c|}
\hline $\begin{array}{l}\text { Atom } \\
\text { Type }\end{array}$ & Category & Atom Id \\
\hline 1 & CM- "Alkene $\mathrm{RH}-\mathrm{C}=$ " & $1,2,150,151$ \\
\hline 2 & CT1- "Alkane >CH-“ & $3,4,27,80,133,152,153$ \\
\hline 3 & CT0- "Alkane -CH2-“ & 7,156 \\
\hline 4 & C- "Imide C=O" & $\begin{array}{l}8,9,45,46,66,67,98,99,119,120, \\
157,158\end{array}$ \\
\hline 5 & HC- "Alkene H-C=" & $11,12,160,161$ \\
\hline 6 & HC1- "Alkane H-C" & $\begin{array}{l}13,14,15,16,17,18,38,39,91,92, \\
144,145,162,163,164,165,166, \\
167\end{array}$ \\
\hline 7 & O- "Imide C=O" & $\begin{array}{l}19,20,53,54,69,70,106,107,122, \\
123,168,169\end{array}$ \\
\hline 8 & N- "Imide -NH-“ & $10,44,68,97,121,159$ \\
\hline 9 & CA0- "Benzonitrile C-CN" & $21,31,84,127,137$ \\
\hline 10 & CA- "Aromatic C" & $\begin{array}{l}22,23,24,25,26,28,29,30,32,33, \\
47,48,49,50,51,52,60,61,62,63, \\
64,65,74,75,76,77,78,79,81,82, \\
83,85,86,100,101,102,103,104, \\
105,113,114,115,116,117,118, \\
128,129,130,131,132,134,135, \\
136,138,139\end{array}$ \\
\hline
\end{tabular}




\begin{tabular}{|l|l|l|}
\hline 11 & HA- "Aromatic H-C" & $34,35,36,37,40,41,42,43,55,56$, \\
& & $57,71,72,73,87,88,89,90,93,94$, \\
& & $95,96,108,109,110,124,125,126$, \\
& & $140,141,142,143,146,147,148$, \\
& & 149 \\
\hline 12 & C1- "Benzophenone C=O" & 58,111 \\
\hline 13 & O1- "Ketone C=O" & 59,112 \\
\hline 14 & CT2- "Alkane >CH-“ & 5,154 \\
\hline 15 & CT3- "Alkane >CH-“ & 6,155 \\
\hline
\end{tabular}

Using the same OPLS-AA force field parameters, provided by TINKER, that was used to define the atom types, now the different types of bonds, angles and dihedrals were identified and listed out.

19 Bond types, 35 Angle types and 60 Dihedral types were recognized in a single PMR-15 monomer. Because of the same reasons for introducing two new atom types as mentioned earlier, a new bond type, type 19, was introduced as it is the new bond formed due to crosslinking. Because the new bond formed is again of type 7, i.e. CT1-CT1 (See Appendix A.2), the force field parameters of this particular bond formed was taken to be same as that of type 7 .

The next step was to define the Cartesian positions of each atom. The initial positions of all the atoms were set up in 2-D planes with just $X$ and $Y$ directions, though allowed to move in any direction as the simulation proceeded. Then the force field parameter of each bond, angle and dihedral was identified and listed down in the data file. The single PMR-15 chain was then replicated 150 times to get all the atoms of the 150 chains of PMR-15 in a simulation box of $2000 \times 2000 \times 2000 \AA^{3}$. Here the initial co-ordinates of atoms and bonds were calculated and the center of the molecule was averaged out. Then using the rotation matrix (Arvo, J., 1992) the coordinates around the $x, y$ and $z$ axis were rotated and the molecules that were translated were evenly spaced out throughout the 
specified volume of the simulation box. Once the positions were assigned to these new molecules, their new bonds, angles and dihedrals were exactly replicated from the initial molecule created in the data file. The fashion, in which the PMR-15 monomers were positioned before the initialization of the deformation and crosslinking simulations, is as mentioned below:

X-Axis: 10 monomer chains

Y-Axis: 5 monomer chains

Z-Axis: 3 Monomer chains

By the term positioning of PMR-15 monomers, it is meant that these many numbers of PMR-15 monomers were copied from the initial data file at the $X, Y$ and Z-Axes.

\subsection{Input Script:}

Once the data file was created, an input script was written down to read this data file in the LAMMPS (Plimpton, S., 1995) software with specific energy minimizations to achieve a stable and equilibrated condition of the system at a specific density of 1.3-1.4 g/cc (Scola, D. A., Ployimide Resins, University of Connecticut, 105-119). LAMMPS reads the commands mentioned in the input script, one line at a time. In an input script made to run in LAMMPS, generally the following structure is followed:

1. Initialization: In this section the parameters required to be followed during the simulation are mentioned. Commands like the style of units used, dimensions of the simulation box, type of simulation box, boundary conditions etc. are mentioned.

2. Atom Definition: This means to define the atom properties like atom position, spatial information etc., in the molecule(s) that is used by LAMMPS while executing the simulation. Atom definition can be done by either reading the data from the data file or the restart file using the read_data or read_restart command. 
3. Settings: Once LAMMPS reads the data file, various settings according to the requirement can be defined for LAMMPS to execute the simulations. Some of the common settings specified in this modeling are force field coefficients like pair, bond and angle coefficients, special bonds, number of time steps, fixes etc. Output commands like thermo, dump and restart were also used to set to achieve the required results.

4. Running the Simulation: Finally the input script mentions the run the command which tells LAMMPS to start the simulation of the system and make it run for the mentioned time.

So, at every mentioned time-step, the assigned energy minimization commands bring the whole system to the desired state of equilibrium. Three types of energy minimizing commands were initially used on the system of 150 monomers of PMR-15 to start the simulation with. They were:

- Fix viscous Command: This command adds a damping viscous force to the atoms and acts similar to a frictional force, bringing the velocity and hence the kinetic energy of the atoms to an equilibrium state. The damping force added through this command is proportional to the velocity of the atoms.

- Fix temp/rescale Command: This command does exactly what it says. It rescales the temperature of the system of atoms to the mentioned value. The provision of keeping the temperature of the system in a window from lowest to the highest desired values is also provided by LAMMPS. In this case, a temperature scale of $30^{\circ} \mathrm{K}$ with a window of $30^{\circ} \mathrm{K}$ was specified, i.e., the system were kept around an average temperature of $30^{\circ} \mathrm{K}$. By allowing a window of $30^{\circ} \mathrm{K}$, it was made sure that, if the temperature of the system exceeded $60^{\circ} \mathrm{K}$ or if dropped below $0^{\circ} \mathrm{K}$ was cooled down or heated up accordingly by the minimizers to bring the average back up to the $30^{\circ} \mathrm{K}$ scale. 
- Fix nve/limit Command: In a system of highly overlapping particles, a condition might arise where large amount of forces might be created that could blow the system apart and bring the simulation to an abrupt end. This command makes sure that, this doesn't happen, by limiting the maximum distance an atom can move and also its velocity. By doing so, generation of large forces due to van der Waal's etc. can be prevented.

Because one of the main objectives of this thesis is to model the PMR-15 polyimide for real life applications, it was necessary to make sure that the density of this model is approximately $1.324 \mathrm{~g} / \mathrm{cc}$ (Scola, D. A., Ployimide Resins, University of Connecticut, 105-119). This could be achieved by decreasing the volume of the simulation box till the required density is achieved. The total mass of the 150 monomers of PMR-15 with 25350 atoms of Carbon, Hydrogen, Oxygen and Nitrogen was calculated to be 218923.95 grams/mole. So, to achieve a density of $1.324 \mathrm{~g} / \mathrm{cc}$, a box size of approximately 65 Angstroms was calculated to be required. Decreasing the simulation box size from its original size of $2000 \AA$ to $65 \AA$ at once, would introduce large amount of forces into the system and that might blow up the atoms apart from each other. So, a series of step by step deformation was carried out until the desired density was achieved. This was done by applying the initial deform command to the data file (refer Appendix B.1) by reducing the simulation box size from $2000 \AA$ to $1000 \AA$. But to get a uniform deformation of the box and to avoid any density localization, each deformation size was equally divided from the center of the simulation box. So, the first deform command was specified to -500 to $500 \AA$ in each of the $X, Y$ and $Z$ axes of the box as the final dimension of the simulation box. A restart file, is the output file from the simulation that is used as the data file for the next simulation, so that it can be carried out from the exact point where the preceding run was stopped. The restart file from the first run, was used to deform the simulation box further to $-250 \AA$ to $250 \AA$ on each of the axes of the box. Each of the restart files from the previous run was used to deform the simulation box by approximately half of the previous size, so as to avoid a sudden disruption in the system. This was done till 
$-32.5 \AA$ to $32.5 \AA$, i.e. $65 \AA$ total box length of the cubic simulation box. And this was the dimension at which a density of $1.324 \mathrm{~g} / \mathrm{cc}$ was achieved.

Once the required density was achieved through a series of step-wise deformation commands, the resulting output/restart file, of the system was made to run without any further deformation for one nanosecond. This step was performed to bring the system to a stable condition within the set temperature and viscosity. 


\section{CHAPTER 3: CROSSLINKING OF PMR-15}

The process where bonds are formed to hold long chains of polymers together is defined as crosslinking. These bonds are created due to presence of two or more reactive groups in a polymer, which undergo chemical reaction in the presence of various factors like heat, pressure, catalysts etc. to bond together and form long chains of polymers. Crosslinking can also take place when the molecules are exposed to radiation. In most of the cases, highly crosslinked polymers have been observed to present very good mechanical properties like higher tensile strength, good thermal resistance, decrease in flexibility, fluid resistance, etc. So, it is very necessary for a polymer to have higher crosslinking ratios. Crosslinking ratio can be defined as the ratio between the total numbers of created crosslinking to the maximum number of crosslinking possible in a system of polymers. A $100 \%$ crosslinking ratio is very difficult to achieve because of various chemical and physical limitations. However, a very high rate of crosslinking is also sometimes not preferred due to the fact that, this may cause some of the material to become very brittle and susceptible to cracking at high temperature and pressure.

Generally PMR-15 polyimides with crosslinking of more than $75 \%$ are said to be of high grade quality. For this research, to get a good crosslinking ratio, it was necessary to introduce as many molecules of PMR-15, near each other, as possible. This chapter deals with the crosslinking procedure of PMR-15 at the chemical level and how it was achieved for this model using commands provided by LAMMPS (Plimpton, S., 1995). In this model, a single chain of PMR-15 was replicated to create 150 more chains. 


\subsection{Reverse Diels-Alder Mechanism}

To understand Reverse Diels-Alder mechanism, let's first define Diels-Alder reaction. Reaction between a dienophile and a conjugated diene that results in the formation of a cyclohexene is called as Diels-Alder Reaction (Diels, O.; Alder, K. 1928).

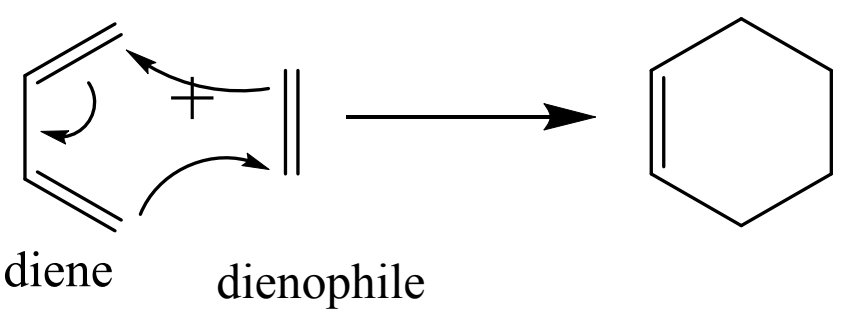

Figure 3.1: Diels-Alder mechanism

Reverse or retro Diels-Alder Reaction is just the opposite of Diels-Alder Reaction, where, under the influence of heat, or with acid/base concentration a cyclohexene group is reacted to form a diene and a dienophile (Rickborn, B., 1998).

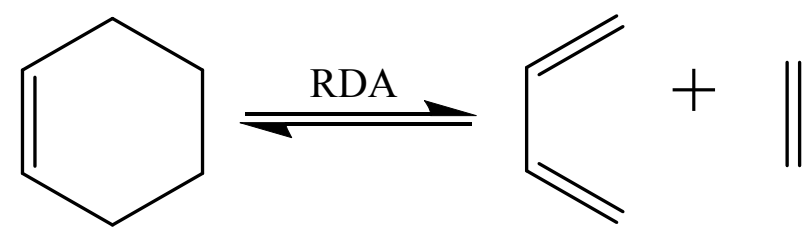

Figure 3.2: Reverse Diels-Alder mechanism

There is a lot of controversy and obscurity regarding the crosslinking reaction of PMR-15. Studies done by NASA, show that the noroborene end-capped imide prepolymer of the PMR-15 chain goes through the reverse Diels-Alder process to produce cyclopentadiene and maleimides which is succeeded by the initiation of chain polymerization. Different analytical instruments, have helped in finding a temperature range of $60-280^{\circ} \mathrm{C}$ in which the imidization of PMR-15 takes place. Using infrared spectroscopy, Parker et al. analyzed the evolution of cyclopentadiene, methanol and water. They found out that the decomposition of ester and 
amine groups, results in the formation of imide and anhydride bands. The formation of cyclopentadiene group begins at $180^{\circ} \mathrm{C}$ and is maximum at $260^{\circ} \mathrm{C}$ (Parker et al., 1990).
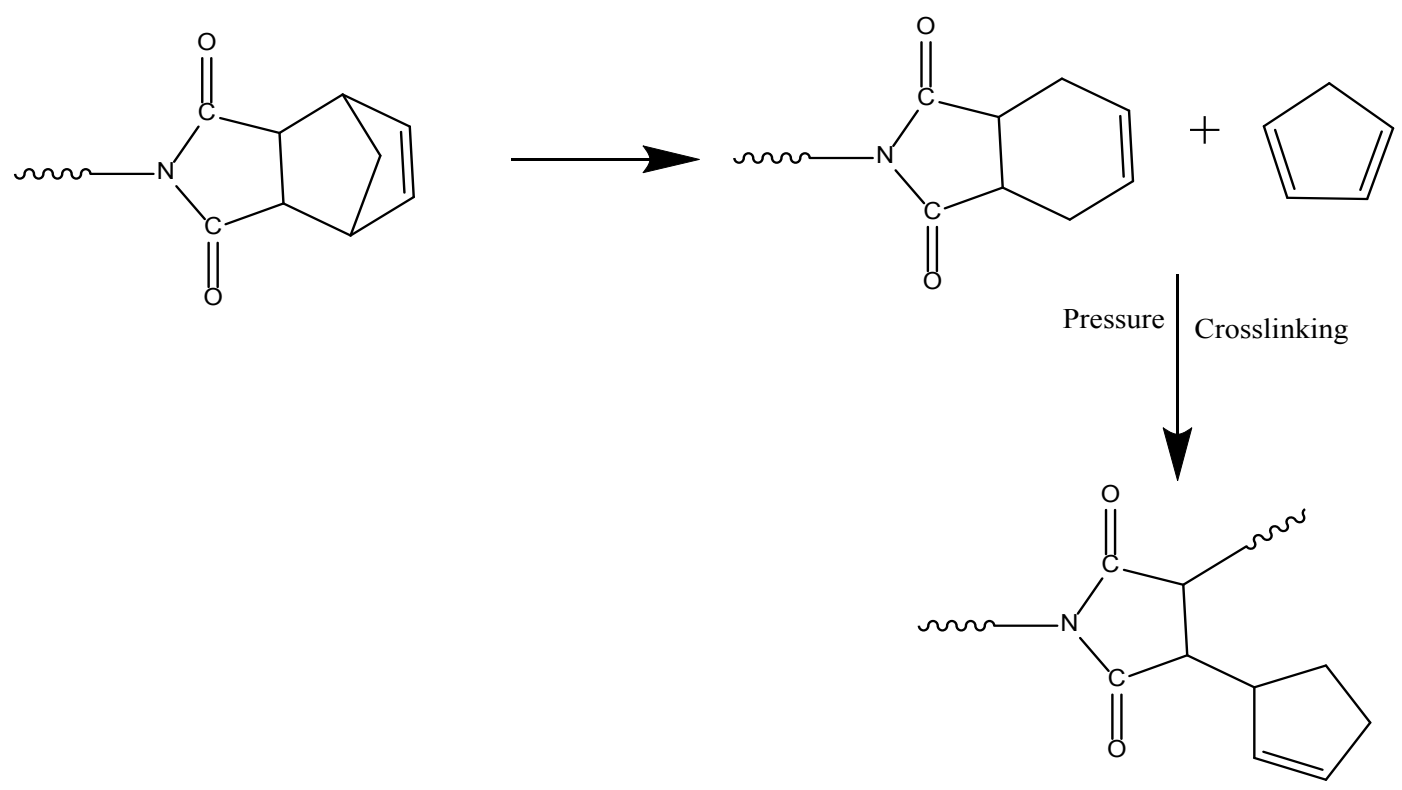

Figure 3.3: Reverse Diels-Alder Reaction of the Noroborene end-caps

BTDE carbonyl containing groups of imide, amide, anhydride, ester, salt and acid converts up to $70 \%$ of imide form at $240^{\circ} \mathrm{C}$. But, the non-imidized forms of BTDE with the free terminals in the MDA group are said to be the cause of transgression in the polymerization reaction. Studies by Preston et al., using dynamic mechanical and thermal analyzer (Preston et al., 1989) have suggested that, increasing the BTDE amount, increases the rigidity of the matrix by providing stronger chain extensions. Though the modulus increases in this process, the glass transition temperature decreases and thermal degradation increases. 
Assumption:

1. Under the given pressure and temperature conditions, all the noroborene end-caps of the 150 PMR-15 chains undergo reverse Diels-Alder reaction to break into cyclopentadiene and maleimide groups.

2. All the carbon atoms with a free radical in the cyclopentadiene and maleimide group within a distance of $7 \AA$, undergoes free-radical polymerization to crosslink with each other. This value of $7 \AA$ is considered based on the facts that a range of 5-10 A has been proven to be effective as a general cut-off distance in achieving good crosslinking percentages in various case of epoxy modeling (Bandyopadhyay, A., 2012; Bandyopadhyay et al., 2012; Bandyopadhyay, A., 2011). So, in order to avoid problems like getting very low crosslinking ratio or to get very high van der Waal's energies among the atoms etc., a value of $7 \AA$ was chosen.

\subsection{Crosslinking Command}

Crosslinking of the 150 chains was carried out by using the Fix bond/create command. This command enables the creation of bonds between atoms to cross-link the polymers. Using this command, bond was created between the carbon atoms of Atom Types 14 and 15 (as mentioned above in Table 1.1) if they were within a distance of $7 \AA$. These carbon atoms were allowed to form a bond with only one other carbon atom of either 14 or 15 atom type. Once the bond was created between any two carbons of 14 and 15 type, these atoms exhibit the same property as the carbon of atom type 2 . So the bond formed, was designated the same bond type as that of the bond type between two atom type 2 carbons. The command used is given below:

"fix 5 all bond/create 514157.019 iparam 12 jparam 1 2" (Appendix B.2) here,

- "5 all" is the ID and the group-ID respectively 
- "bond/create" is the style name of the fix command

- " 5 " = attempt bond creation every this many steps

- "14 15" = atoms of itype can bond to atoms of jtype

- "7.0" = two atoms separated by less than minimum cut-off distance can bond (distance units)

- "19" = type of created bonds

- "iparam 12 " = Parameters of itype atom - maximum number of bonds of bondt ype the 14 atom types can have; change the 14 atom types to this type when maximum bonds exist

- "jparam 12 2" = Parameters of jtype atom - maximum number of bonds of bond type the 15 atom types can have; change the 15 atom types to this type when maximum bonds exist

\subsection{Density Distribution}

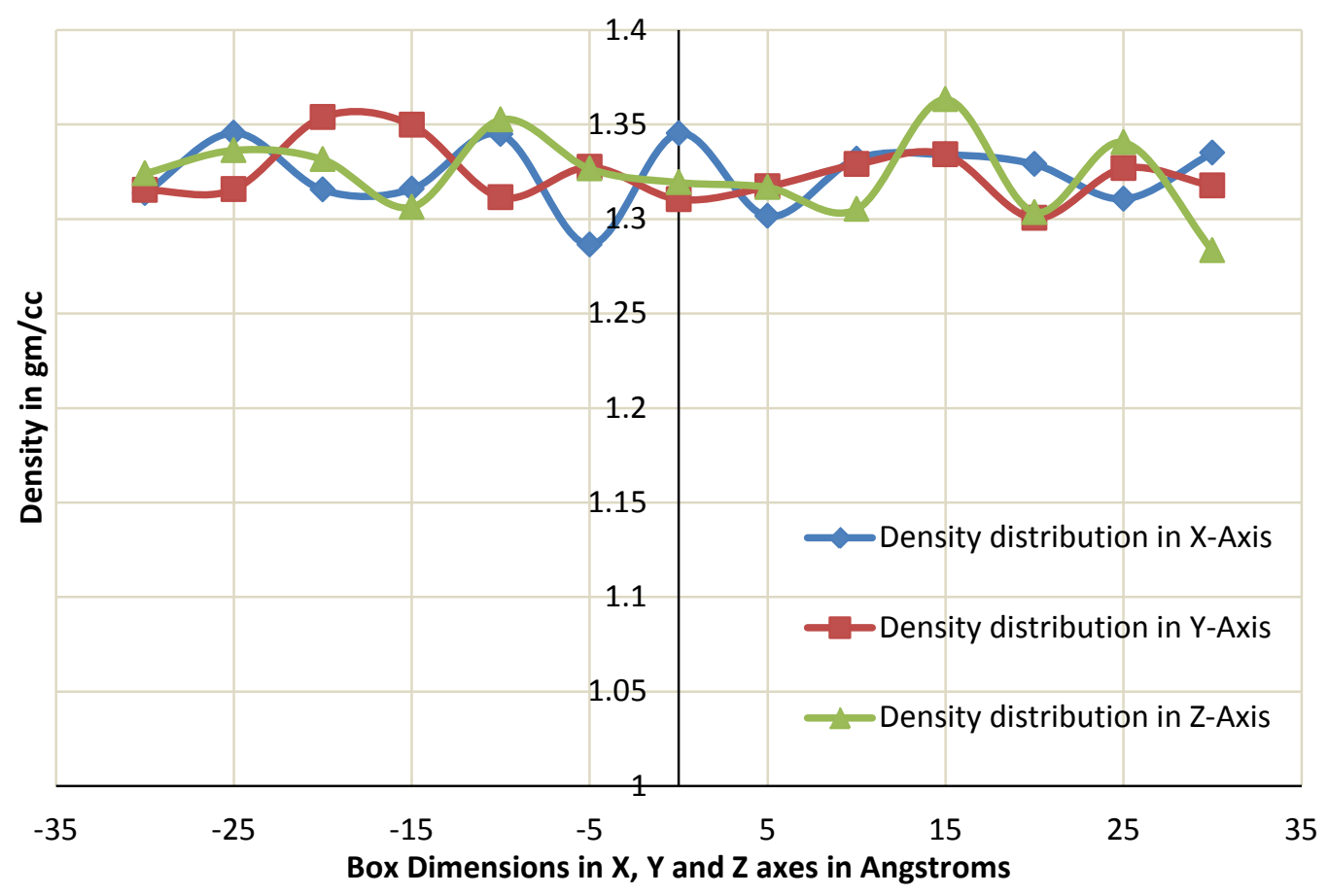

Figure 3.4: Density distribution chart along $X, Y$ and $Z$ Axes 
To determine if there was any localized density in the simulation box, the spatial density distribution was calculated using the Fix ave/spatial command. To achieve this, 15 equal divisions were made along the $X, Y$ and $Z$ axes of the final simulation box. The masses of the atoms present in each of these divisions were determined which was then used to determine the density by dividing it by the volume of the divisions.

The results of this were plotted in a graph and as shown in Figure 3.1. From the Figure 3.1 it is clear that the density distribution along the $X, Y$ and $Z$ axes of the simulation box were uniform. The reason for small non-uniformity in the system could be attributed to the fact that some of the divisions contained more atoms than the others due to high number of crosslinking chains in them. The large number of crosslinking chains, reduce the mobility of atoms in the system and give rise to compressive forces, high enough to restrict the molecular movement and to produce large local densities. Other than severing the uniformity of the density distribution, the high crosslinking percentage also challenges the prediction of physical properties of the system. 


\section{CHAPTER 4: RESULTS AND CONCLUSION}

The 150 chains of PMR-15 were made to run under NVE (constant Volume and Energy) simulations at a temperature range of $1 \mathrm{~K}$ to $300 \mathrm{~K}$ with constantly increasing the density of the model by decreasing the simulation box size. This process of step wise decreasing the simulation box size to achieve the density, took a total of about 55 nanoseconds. The high range of temperature allowed the system to release the energy in the form of heat whenever it underwent high compressive forces due to deformation. Upon the constant deformation of the simulation box to achieve a density of $1.324 \mathrm{~g} / \mathrm{cc}$ and after performing the crosslinking of the chains as discussed in the above section, the final system is as shown in Figure 4.2. The following sections discusses about how the final system of 150 crosslinked chains of PMR-15 were made to run under NVT and NPT commands to validate the model for its real time use. An attempt to bring down the system to a steady state with its parameters close to those of ambient working conditions was made by running a NVT simulation for 10,000 picoseconds with a temperature range of $300 \mathrm{~K}$ (Appendix B.4). The time selected for the simulation to run, provides us with ample data to verify if the system can survive the natural working conditions and is stable in that environment. The main objective of performing the NVT simulation was to bring the pressure of the system down to a minimum of approximately $1.0 \mathrm{~atm}$. After this the system was made to run under NPT simulations for 1000 picosecond (Appendix B.5). The temperature was allowed to damp at every 1000 femtoseconds and the pressure was allowed to damp at every 5000 femtoseconds. By damping, it is meant that, the temperature or the pressure was not allowed to increase or decrease instantly, but was let to relax to the mentioned time units until the minimizer would act again to bring the temperature/pressure to the desired range. This would keep the system in a stable condition even when high amount of energy was introduced or removed from it. 


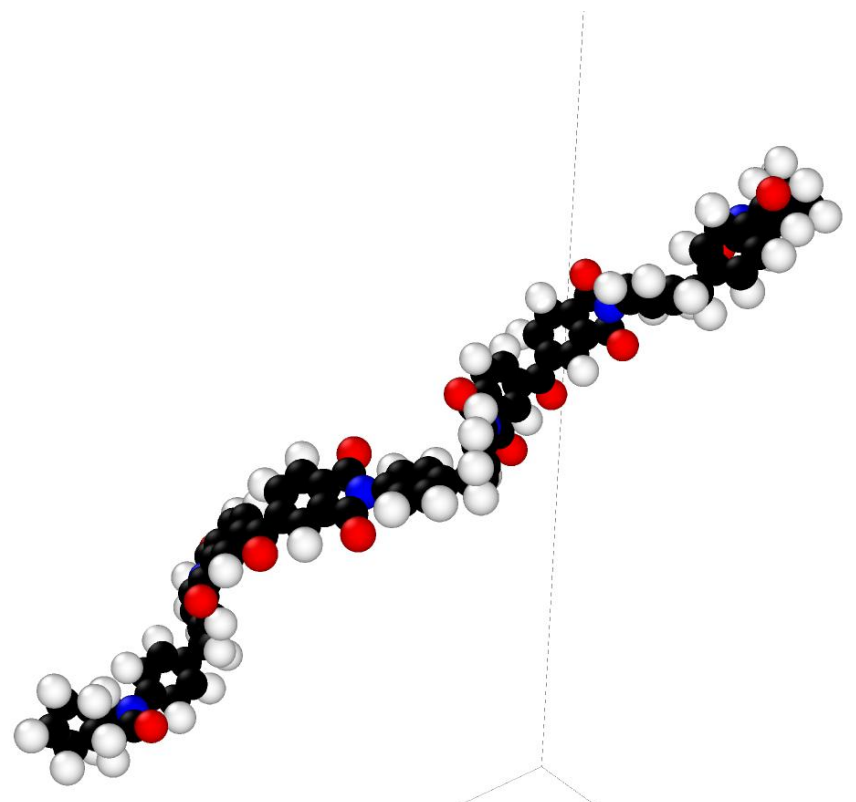

Figure 4.1: Structure of a single PMR-15 chain in the $2000 \times 2000 \times 2000$ $\AA^{3}$ simulation box

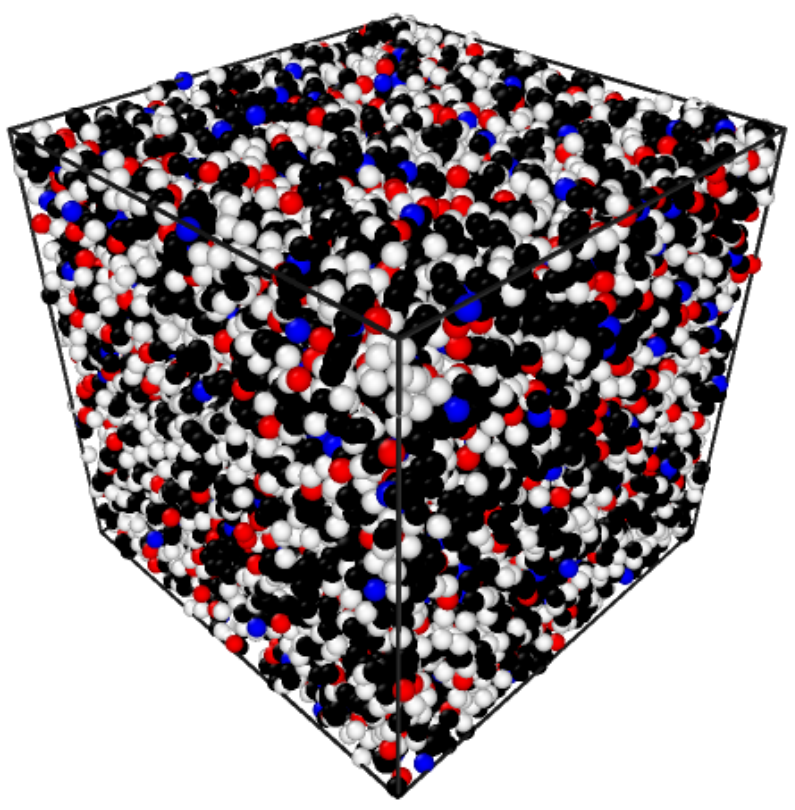

Figure 4.2: PMR-15 model of 150 chains crosslinked together at a density of $1.324 \mathrm{~g} / \mathrm{cc}$ 


\subsection{Time versus Pressure}

To check if the model is correct, and can be used in future for simulating under different conditions, it is very important to verify if the model retains the same properties, when modeled, as in ambient conditions. When the model was created, and after the deformation as well as the create bond commands were implemented (Appendix B.1 and B.2), it was observed that the pressure in the system was little higher than the normal conditions, i.e., in the range of 35000$40000 \mathrm{~atm}$. The output data from this system was made to undergo NVT simulations over a period of 10000 picoseconds (Appendix B.4). From the graph as shown in Figure 4.3, we can observe that the pressure, during the crosslinking, fluctuates around 35500 to $40500 \mathrm{~atm}$. As NVT simulation was run on the model, the range of pressure drops down, but not considerably. Because of the nature of NVT ensemble, only the temperature of the system was changed to the mentioned range, keeping the volume constant. The pressure of the system was allowed to fluctuate. The next step was to bring down the pressure to validate the equilibrium state. This was done by making it run under the NPT command (Appendix B.5). NPT ensemble, also called as isothermal-isobaric ensemble, as the name suggests, was used over a period of approximately 1000 picoseconds to get the pressure around $1.0 \mathrm{~atm}$. And that's the reason why we find a sudden drop in the pressure of the system after 10,000 picoseconds. 


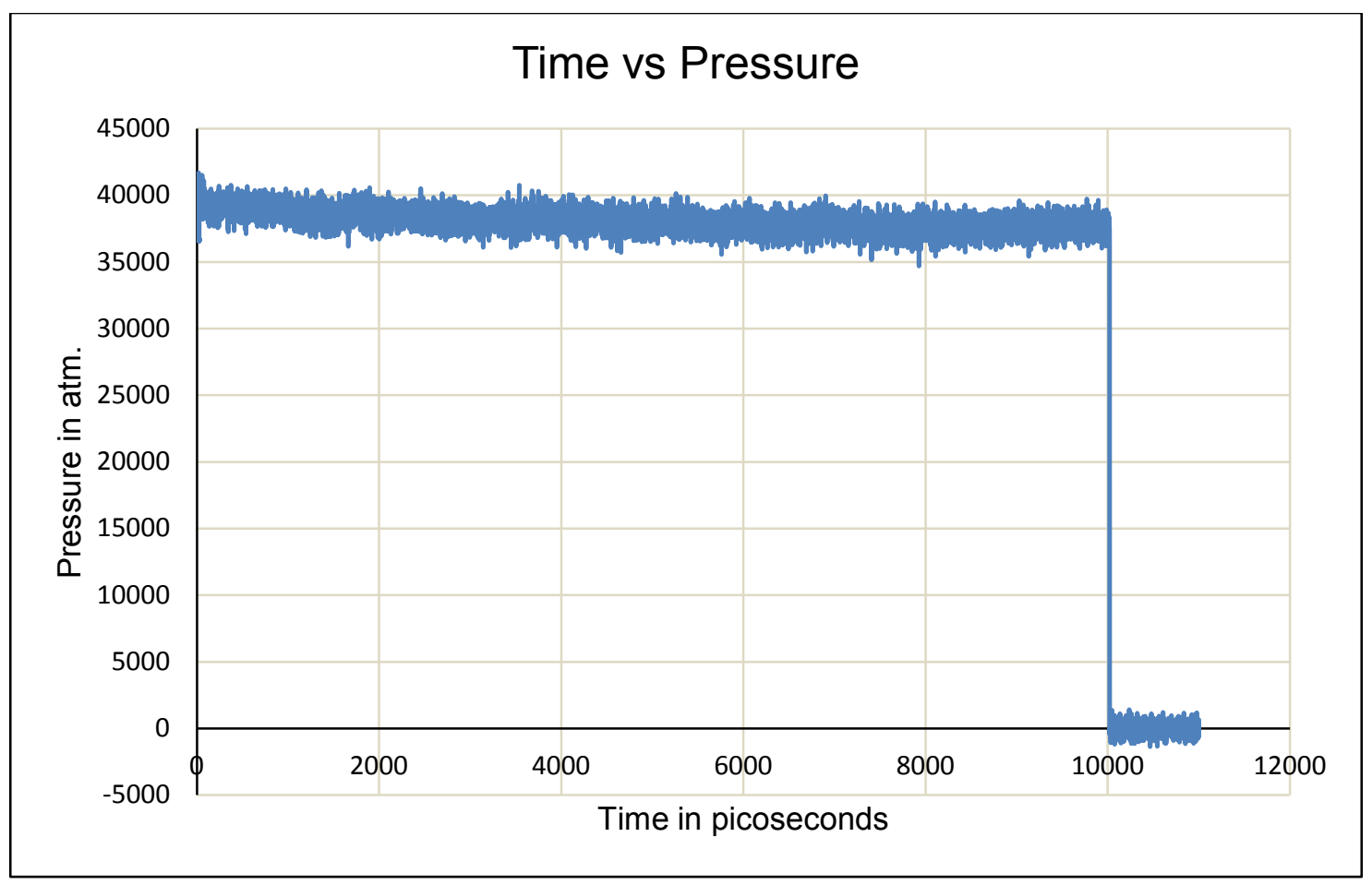

Figure 4.3: Time vs Pressure

\subsection{Pressure versus Density}

Even though the system doesn't show any errors, while existing under the pressure of around $1.0 \mathrm{~atm}$., it is not necessary that the density set for it remains the same. To verify if the density of the system fluctuates under the NVT command, a graph of pressure versus density was plotted. From this plot in Figure 4.5, it can be seen that, under the pressure of 35000-40000 atm., i.e., the pressure in which the system was initially modelled, the density remains to be around pre-set value of $1.324 \mathrm{~g} / \mathrm{cc}$. But as the pressure was dropped using NVT command, it was observed that the density also drops to a value of $0.9467 \mathrm{~g} / \mathrm{cc}$. The reason for this drop of density could be due to the fact that, the rate at which the pressure was dropped, was so sudden that, the simulation box of the model had to expand a little to prevent the system from coming to an abrupt stop in its simulation. Because the deform command was not applied during this course of simulation, the box was free to expand to achieve the desired pressure for the 
system. Even then, the change in density of the polyimide was calculated to have a difference of $28.5 \%$ only.

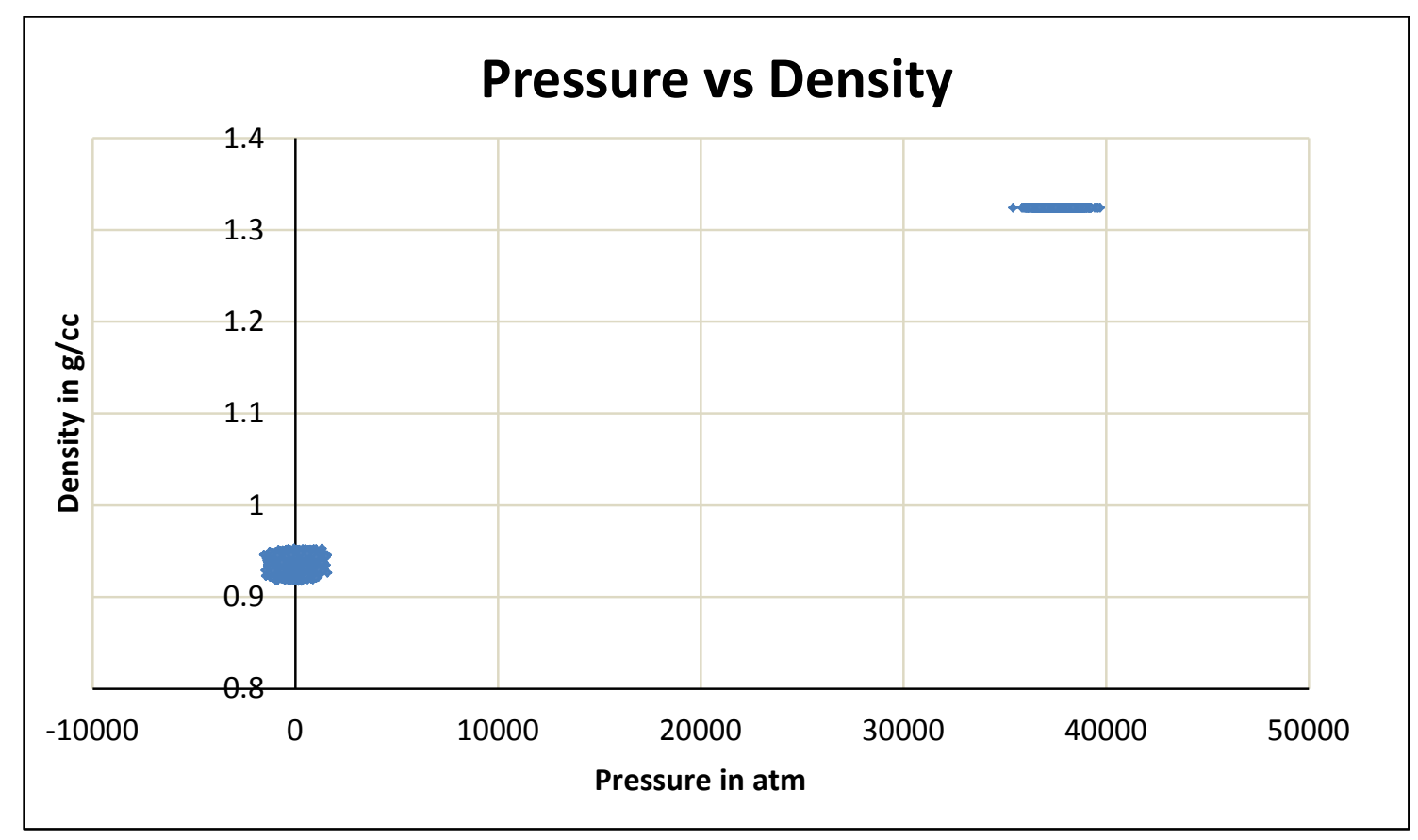

Figure 4.4: Pressure vs Density

\subsection{Time versus Potential Energy}

Another factor which validates the equilibrated condition of the modeled system is the plot between time and the potential energy. For a given system it is necessary that the potential energy be as minimum as possible. We can see from Figure 4.5 that, the potential energy of the system is extremely high during the initial simulations of the model. Applying NVT command as well as the energy minimization commands on the densified and crosslinked system over 10 picoseconds considerably brings down the potential energy of the system. The reason for this drop down is because the system acts under the energy minimizers and NVT. In this, the ensemble provides each particle in the system a different position than its original position. This is done under constant Volume and Temperature (NVT). It then calculates the potential energy and the net forces acting on the particle due to its trail position. Highly complicated mathematical 
formulas and terms are used to calculate these potential energy and the net forces on these particles. Under the energy minimization method, if the new energy of the system due to the trial position is within the limits and also, if the net forces acting on the particle is equal to zero, then the trial position of the particle is saved as its new position. But if the net forces are not reached to zero, then that particular trial position is not considered and a new trial position is provided to the particle. This cycle continues until the net forces on each particle is zero. What this theory shows that is, on applying energy minimization and NVT simulation, the energy of the particle has to come down because the new position can be considered only if the net forces on the particles is equal to zero. So, the particles are kept on moving to new positions until the energy of the whole system comes down. That is the reason why we see such a drastic drop in the potential energy of the system. The same applies to NPT ensemble too. That is the reason we see a further drop in the potential energy after 10.02 picoseconds when the NPT simulation takes over from the NVT simulation. 


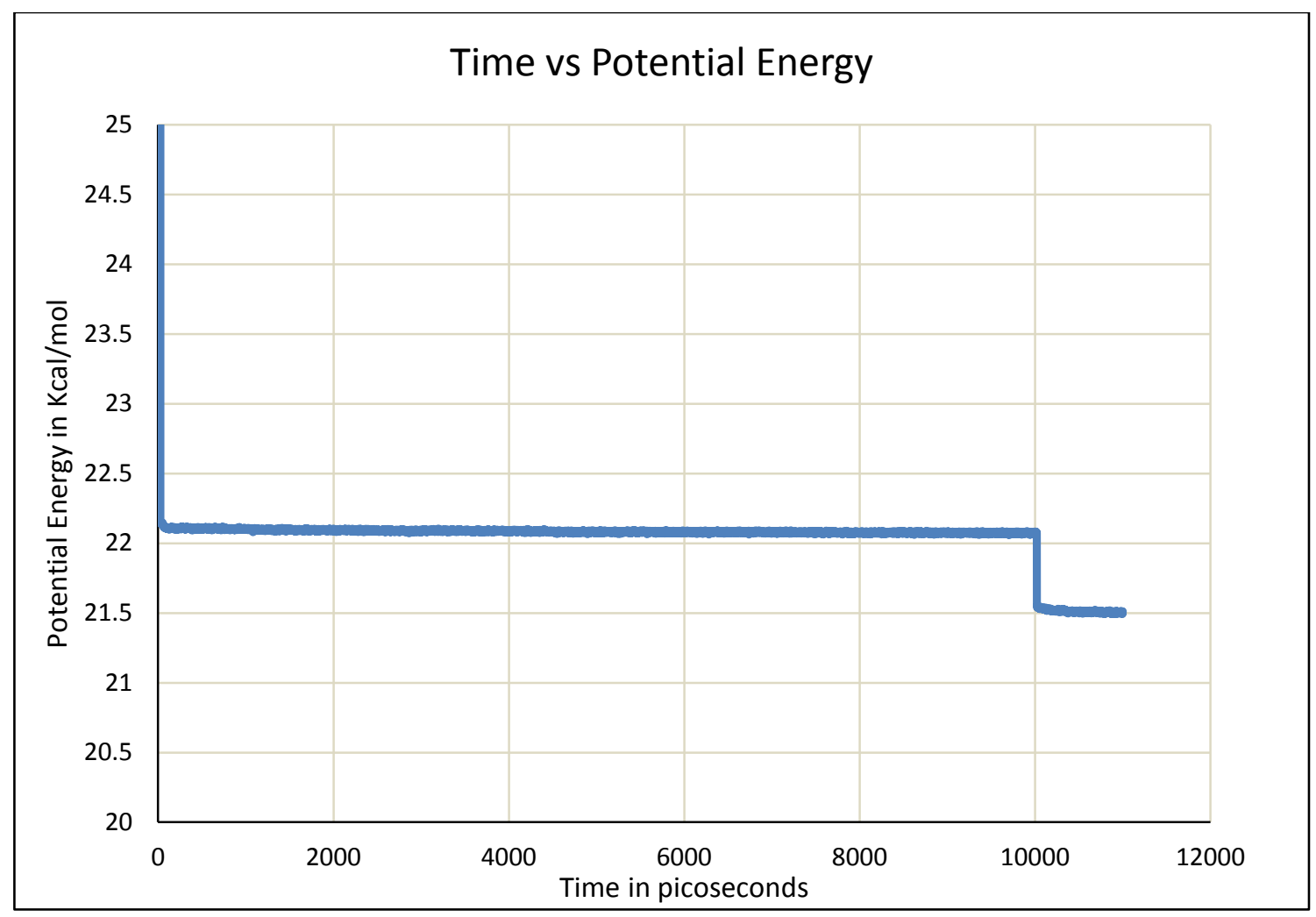

Figure 4.5: Time vs Potential Energy

\subsection{Time versus Kinetic Energy}

The Kinetic energy of the system here is calculated and plotted with respect to time as shown in Figure 4.6. From Figure 4.6, we can see that the kinetic energy of the system comes down as soon as the NVT simulation is applied and continues to remain under a given range till the end of the simulation and even when NPT was applied. This suggests that the kinetic energy is not changing drastically, and is at an equilibrated condition throughout. 


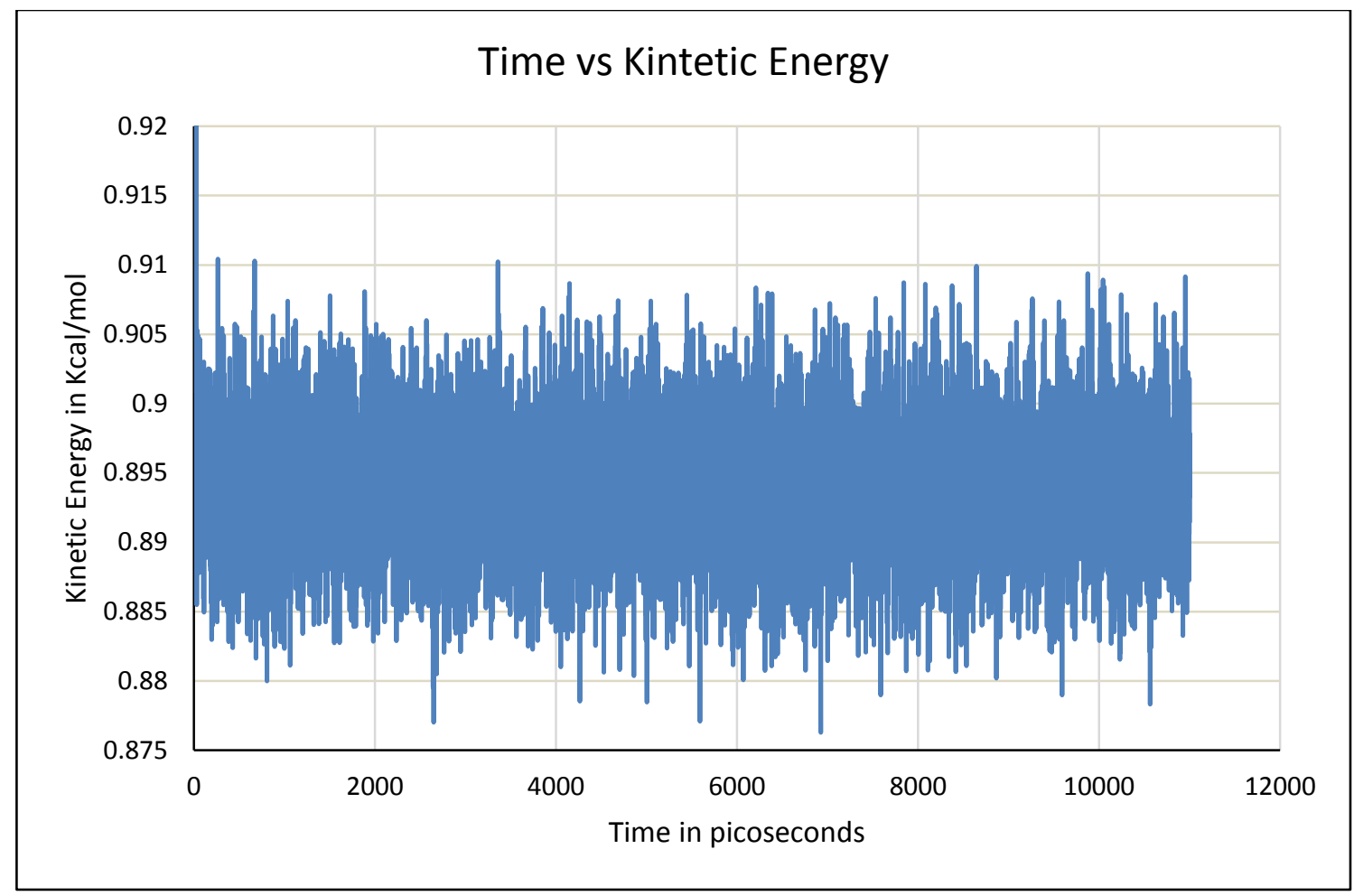

Figure 4.6: Time vs Kinetic Energy

\subsection{Time versus Energy}

Going back to equation:

$$
E(\phi)=E_{\text {bond }}(\phi)+E_{\text {angle }}(\phi)+E_{\text {non-bonded }}(\phi)+E_{\text {torsion }}(\phi)
$$

We can say that, this function of potential energy is the sum of energy terms that define the change in the bond lengths, bond angles, torsion angles and van der Waals values from the equilibrium state. Again in this case, the energy minimizers come into action and optimize these energies to a minimum level. This is also done by performing calculations at the quantum level to achieve lower energy states. These lower energy states are more desirable due to their stability and hence are preferable in performing studies at equilibrated conditions. Figure 4.7 shows how the implementation of NVT command and then of NPT command decreases the total energy of the system by bringing down the bond energy, bond angle energy, torsional angle energy, and van der Waal's energy. 


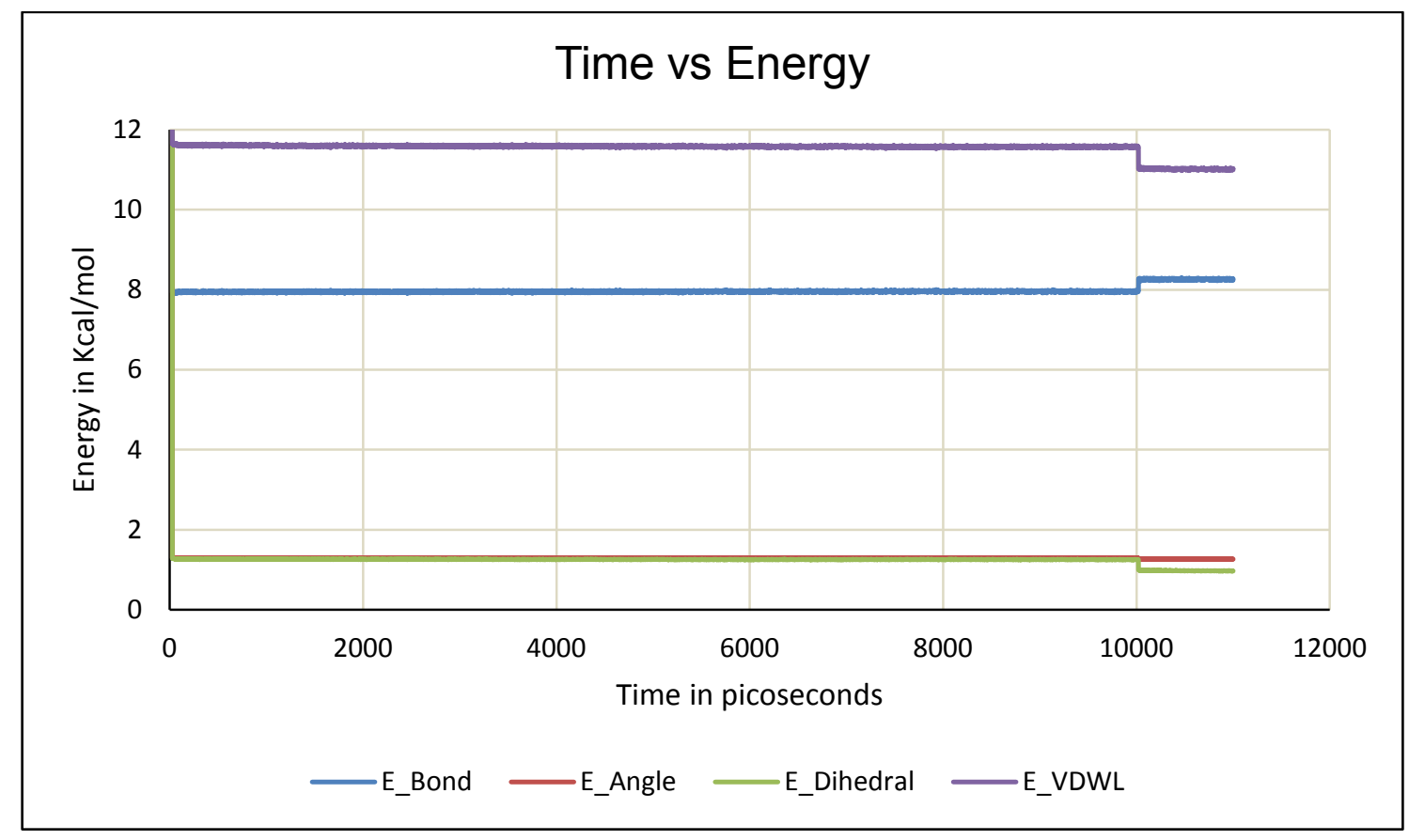

Figure 4.7: Time vs Energy

\subsection{Conclusion}

This research has modeled and analyzed the structure of PMR-15 polyimide with 25350 atoms and a given density of $1.324 \mathrm{gm} / \mathrm{cc}$ using OPLS-AA force field parameters. The tests for validation conducted under the NVT and NPT command and the above mentioned results prove that the model is able to withstand the sudden drop of pressure and potential energy or increase in temperature. However, the biggest limitation of this model lies in the fact that the density of the system does not remain constant throughout. This can be observed from the results discussed in Section 4.1 and 4.2. As the NVT and NPT simulations were run on the model, it was observed that the pressure of the system drops to a considerable and desirable value. But when the density plot with pressure was plotted (Section 4.2), it was seen that the density of the model decreases. This shows that, it was unable to maintain the corresponding density of the material, and the model failed to remain stable. The reason for this failure is because of 
the use of OPLS force field parameters. Using OPLS-AA force field parameters, does not validate the system of molecular model to remain stable and show a consistent density. So, it can be concluded that using OPLS force field parameters for modeling PMR-15 polyimide should not be considered on the grounds of its instability, for further research on validating its properties. This model can be used as reference to develop better and more equilibrated MD models of crosslinked PMR-15 molecules which can eliminate these drawbacks. Also, different modeling techniques can be used in future to validate the mechanical properties. 


\section{CHAPTER 5: FUTURE SCOPE}

Molecular Dynamic though being a fairly new technique in the field of computational research, it has developed as a very important tool in understanding the physical phenomenon of systems at the molecular level. MD has been used extensively to study the behavioral patterns of polymers in general. A lot of research has been done on PMR-15, but still the study on the aging of the polyimide and the resulting changes in its mechanical and physical properties are pending. Using this model, an attempt to answer these questions can be made by simulating the models under different loading and aging conditions. The variations, if any, in the properties of PMR-15 at different crosslinking ratios, environmental conditions, etc. can also be easily determined in much cheaper, easier and faster ways.

\subsection{Development}

The major concern regarding PMR-15 is the presence of its toxic and carcinogenic constituent MDA, which makes its processability very difficult and costly affair. When first evaluated, PMR-15 was predicted to be high on demand by the year 2000. But the mutagenicity of MDA (US Dep. Health Hum. Serv., 1986) and the costs involved in providing safer production conditions, prevented it from being a major success (Meador 1998). A lot of research has been done in the past to find a method to develop materials similar to PMR-15 without containing MDA as a constituent. This model can help in analyzing the molecular structure of PMR-15 that governs the desirable properties of this polyimide so that it could be recreated in the required manner. 


\subsection{Aging}

A lot of research has been done in the aging process of PMR-15 and the resulting degradation of the polyimides. Using MD can make way to perform such experiments in much faster ways. Some of the types of aging mechanisms occurring commonly in PMR-15 that could be analyzed easily in MD are (Tandon et al.):

5.2.1 Physical Aging: This type of aging results in increased strain and damage the material, primarily because of the decrease in the mobility of the molecules and hence is thermodynamically reversible.

5.2.2 Chemical Aging: Chemical reactions like hydrolysis, depolymerization, additional crosslinking and plasticization are the cause of this type of aging.

5.2.3 Thermo-oxidative aging: Oxidation reactions that reduces the molecular weight of the polymer and leading to surface diffusion are called as Thermo-oxidative aging.

5.2.4 Mechanical stress-induced aging: This type of aging is the one with the least knowledge and least researched by the scientists working on PMR15. This type of aging is caused by the mechanical and thermal fatigue loading. These loadings leads to the growth of micromechanical damage in the material and aggravates the creep development as well as the thermo-oxidative reactions.

\subsection{Failure Properties}

Because of its wide spread use in the aerospace industry, the failure properties of PMR-15 should be studied extensively. But still, there are very few studies done in this field, especially the fracture and creep properties (Odegard, G. M. 2000). Studies have confirmed that during the imidization and crosslinking reaction of PMR-15, voids and bubbles are formed in the polymer. These voids and bubbles are said to be the reason for the adverse effects on the mechanical properties of the material (Farouk, Langrana et al. 1992). MD simulations can be effectively used to determine the cause for the formation of these bubbles and voids. Also, the advantage of such a microscopic analysis is that, even the areas 
or spots in which these deformations arise, can be detected. These results can be used to structure the matrix material in a way to avoid these voids to a minimum.

\subsection{Modeling}

Future modeling of PMR-15 can be undertaken using different force field parameters like those of REACX, which have been proven to be highly effective in many cases. Also, an important factor that can be considered during modeling of PMR-15 is to implement different types of possible crosslinking mechanism of PMR-15. Studies done at BP and Rolls-Royce have confirmed that, the rate of reverse Diels-Alder (RDA) reaction governs the crosslinking mechanism in PMR15 (Hay, Boyle et al. 1989). These studies have shown that the high temperatures, increases the rate of RDA reaction resulting in different type crosslinking mechanism than the type used in this research. Even though characterizing the types of crosslinking is difficult, and no absolute confirmations on the postulated results have been stated, these approach of crosslinking mechanisms are also suggested to be examined in future work. 


\section{REFERENCE}

1. Dangarwala, G. A., POLYIMIDE PMR-15 DURING CURE, Ohio University, (1993).

2. Bandyopadhyay, A., "Molecular modeling of EPON 862-DETDA polymer ", Theses and Dissertations. Paper 21, 2012.

3. Hay, J. N., et al. (1989). "Polymerization of N-phenylnadimide: a model for the crosslinking of PMR-15 polyimide." Polymer 30(6): 1032-1040.

4. Tito, T.; Serafini, Delvigs, P.; Lightse y, G. R., Thermally Stable Polyimides from Solutions of Monomeric Reactants, J. App. Poly. Sci., Vol 16, 905-15, 1972.

5. S. Plimpton, Fast Parallel Algorithms for Short-Range Molecular Dynamics, J Comp Phys, 117, 1-19, 1995, http://lammps.sandia.gov.

6. Diels, O.; Alder, K. (1928). "Synthesen in der hydroaromatischen Reihe". Justus Liebig's Annalen der Chemie 460: 98-122.

7. Parker, S.F.; Hoyle, N. D.; Walton, J. R., The Cure Cycle of PMR-15 as Studies by in-situ Infrared Spectroscopy, High Perf. Poly., Vol. 2, No. 4 , 267-75, 1990

8. Preston, P. N.; Soutar, I.; Woodfine, B.; Hay, J. N., Investigation of the Curing Mechanism in PMR Polyimide Resin, High Perf. Poly., Vol. 1, No. 2, 161-175, 1989

9. Bandyopadhyay, A., G.M. Odegard, "Molecular Modeling of Crosslink Distribution in Epoxy Polymers", Modeling and Simulation in Materials Science and Engineering, Vol. 20, no. 4, 045018, 2012)

10. Rickborn, B., The Retro-Diels-Alder Reaction, Part I, C-C Dienophiles, Org. React. 1998, 52, 1-393.

11. Rickborn, B., The Retro-Diels-Alder Reaction. Part II, Dienophiles with One or More Heteroatom, Org. React. 1998, 53, 223-629. 
12. Jones, David C., "Nanomechanical Characterizations of High Temperature Polymer Matrix Composite Resin: Pmr-15 Polyimide" (2009). University of Kentucky Master's Theses. Paper 595

13. US Dep. Health Hum. Serv., Current Intelligence Bull. 47, Methylenedianiline, July 28, 1986. Washington, DC: USDHHS

14. Tandon, G.P., Pochiraju, K.V., Schoeppner, G.A., Modeling of oxidative development in PMR-15 resin, Polymer Degradation and Stability, Volume 91, Issue 8, August 2006, Pages 1861-1869, ISSN 0141-3910.

15. Odegard, G. M., Shear-dominated biaxial failure analysis of polymermatrix composites at room and elevated temperatures, University of Denver, 2000, ISBN 9780599790162, p. 198

16. Farouk, A., et al. (1992). "Modulus prediction of a cross-ply fiber reinforced fabric composite with voids." Polymer Composites 13(4): 285-294.

17. Hay, J. N., et al. (1989). "Polymerization of N-phenylnadimide: a model for the crosslinking of PMR-15 polyimide." Polymer 30(6): 1032-1040.

18. Hiemenz, P. C. and T. P. Lodge (2007). Polymer Chemistry, CRC PressINC.

19. Bandyopadhyay, A., "Computational prediction of the influence of crosslink distribution on the thermo-mechanical properties of epoxies" (2011). Theses and Dissertations. Paper 349.

20. Jorgensen, W. L., et al. (1996). "Development and Testing of the OPLS All-Atom Force Field on Conformational Energetics and Properties of Organic Liquids." Journal of the American Chemical Society 118(45): 1122511236.

21. Jorgensen, W. L. and J. Tirado-Rives (1988). "The OPLS [optimized potentials for liquid simulations] potential functions for proteins, energy minimizations for crystals of cyclic peptides and crambin." Journal of the American Chemical Society 110(6): 1657-1666. 
22. Meador, M. A. (1998). "Recent advances in the development of processable high-temperature polymers." Annual Review of Materials Science 28: 599-630.

23. Odegard, G., Searles, K., Kumosa, M., "Nonlinear Analysis of Woven Fabric-Reinforced Graphite/PMR-15 Composites under Shear-Dominated Biaxial Loads", Mechanics of Composite Materials and Structures, Vol. 7, Iss. 2, 2000

24. J W Ponder and F M Richards J. Comput. Chem. 1987, 8, 1016-1024.

25. Arvo, J., "Fast random rotation matrices", in David Kirk, Graphics Gems III, San Diego: Academic Press Professional, pp. 117-120, 1992, ISBN 978-012-409671-4

26. Odegard, G. and M. Kumosa (2000). "Elastic-plastic and failure properties of a unidirectional carbon/PMR-15 composite at room and elevated temperatures." Composites Science and Technology 60(16): 2979-2988.

27. Serafini, T. T., et al. (1972). "Thermally stable polyimides from solutions of monomeric reactants." Journal of Applied Polymer Science 16(4): 905915.

28. Odegard, G., K. Searles, M. Kumosa. "A Continuum Elastic-Plastic Model for Woven Fabric/Polymer Matrix Composite Materials Under Biaxial Stresses," Composites Science and Technology, Vol. 61, no. 16, pp. 2501-2510 (2001)

29. Zhigilei, L., University of Virginia, MSE 4270/6270: Introduction to Atomistic Simulations

30. Metropolis, N. (1953). "Equation of State Calculations by Fast Computing Machines." The Journal of chemical physics 21(6): 1087.

31. Sadus, R. J., Centre for Molecular Simulation, Swinburne University of Technology. 
32. Hammond, B.J.; W.A. Lester \& P.J. Reynolds (1994). Monte Carlo Methods in Ab Initio Quantum Chemistry. Singapore: World Scientific. ISBN 981-02-0321-7. OCLC 29594695 


\section{Appendix A}

List of tables of the data file, detailing the Atom mass, Bond Coefficients, Angle Coefficients and Dihedral Coefficients.

Table A.1: Atomic Mass

\begin{tabular}{|l|l|l|}
\hline Atom ID & Mass & Atom Type \\
\hline 1 & 12.011 & \#CM \\
\hline 2 & 12.011 & \#CT1 \\
\hline 3 & 12.011 & \#CT0 \\
\hline 4 & 12.011 & \#C \\
\hline 5 & 1.008 & \#HC \\
\hline 6 & 1.008 & \#HC1 \\
\hline 7 & 15.999 & \#O \\
\hline 8 & 14.007 & \#N \\
\hline 9 & 12.011 & \#CA0 \\
\hline 10 & 12.011 & \#CA \\
\hline 11 & 1.008 & \#HA \\
\hline 12 & 12.011 & \#C1 \\
\hline 13 & 15.999 & \#O1 \\
\hline 14 & 12.011 & \#CT2 \\
\hline 15 & 12.011 & \#CT3 \\
\hline
\end{tabular}


Table A.2: Bond Coefficient

\begin{tabular}{|l|l|l|l|}
\hline $\begin{array}{l}\text { Bond } \\
\text { Type }\end{array}$ & $\mathbf{K}_{\mathbf{r}}$ & $\mathbf{r}_{\text {eq }}$ (Bond Length) & Bond Pairs \\
\hline 1 & 549 & 1.34 & \#CM=CM \\
\hline 2 & 317 & 1.51 & \#CM-CT1 \\
\hline 3 & 340 & 1.08 & \#CM-HC \\
\hline 4 & 340 & 1.09 & \#CT1-HC1 \\
\hline 5 & 317 & 1.522 & \#CT1-C \\
\hline 6 & 268 & 1.529 & \#CT1-CT0 \\
\hline 7 & 268 & 1.529 & \#CT1-CT1 \\
\hline 8 & 340 & 1.09 & \#CT0-HC1 \\
\hline 9 & 570 & 1.229 & \#C=O \\
\hline 10 & 490 & 1.335 & \#C-N \\
\hline 11 & 469 & 1.4 & \#CA0=CA \\
\hline 12 & 469 & 1.4 & \#CA=CA \\
\hline 13 & 367 & 1.08 & \#HA-CA \\
\hline 14 & 317 & 1.51 & \#CA-CT0 \\
\hline 15 & 427 & 1.381 & \#N-CA0 \\
\hline 16 & 400 & 1.49 & \#C-CA \\
\hline 17 & 400 & 1.49 & \#CA-C1 \\
\hline 18 & 570 & 1.229 & \#C1=O \\
\hline
\end{tabular}


Table A.3: Angle Coefficients

\begin{tabular}{|c|c|c|c|}
\hline $\begin{array}{l}\text { Angle } \\
\text { Type }\end{array}$ & $\overline{K_{\theta}}$ & $\boldsymbol{\theta}_{\mathrm{eq}}$ (Bond Angle) & Atom types \\
\hline 1 & 70 & 124 & \#CM=CM-CT1 \\
\hline 2 & 35 & 120 & \#CM=CM-HC \\
\hline 3 & 35 & 109.5 & \#CM-CT1-HC1 \\
\hline 4 & 63 & 111.1 & \#CM-CT1-CT1 \\
\hline 5 & 63 & 111.1 & \#СM-СТ1-СТ0 \\
\hline 6 & 35 & 117 & \#HC-CM-CT1 \\
\hline 7 & 37.5 & 110.7 & \#HC1-CT1-CT1 \\
\hline 8 & 37.5 & 110.7 & \#HC1-CT1-CT0 \\
\hline 9 & 35 & 109.5 & \#HC1-CT1-C \\
\hline 10 & 58.35 & 112.7 & \#CT1-CT1-CT1 \\
\hline 11 & 63 & 111.1 & \#CT1-CT1-C \\
\hline 12 & 58.35 & 112.7 & \#CT1-CT0-CT1 \\
\hline 13 & 37.5 & 110.7 & \#CT1-СТ0-НC1 \\
\hline 14 & 58.35 & 112.7 & \#CT1-CT1-CT0 \\
\hline 15 & 33 & 107.8 & \#HC1-CT0-HC1 \\
\hline 16 & 70 & 116.6 & \#CT1-C-N \\
\hline 17 & 80 & 120.4 & \#CT1-C=O \\
\hline 18 & 70 & 126.4 & \#C-N-C \\
\hline 19 & 80 & 122.9 & $\mathrm{HO}=\mathrm{C}-\mathrm{N}$ \\
\hline 20 & 63 & 120 & $\# \mathrm{CA} 0=\mathrm{CA}-\mathrm{CA}$ \\
\hline 21 & 35 & 120 & $\# \mathrm{CA} 0=\mathrm{CA}-\mathrm{HA}$ \\
\hline 22 & 63 & 120 & \#CA $=\mathrm{CA}-\mathrm{CA}$ \\
\hline 23 & 35 & 120 & $\# \mathrm{CA}=\mathrm{CA}-\mathrm{HA}$ \\
\hline 24 & 70 & 120 & \#CA=CA-CTO \\
\hline 25 & 35 & 109.5 & \#CA-CT0-HC1 \\
\hline 26 & 40 & 109.5 & \#СA-СТ0-СА \\
\hline
\end{tabular}




\begin{tabular}{|l|l|l|l|}
\hline 27 & 63 & 120 & \#CA=CA0-CA \\
\hline 28 & 70 & 120 & \#N-CA0=CA \\
\hline 29 & 50 & 121.9 & \#C-N-CA0 \\
\hline 30 & 70 & 115.5 & \#N-C-CA \\
\hline 31 & 80 & 120.4 & \#O=C-CA \\
\hline 32 & 85 & 120 & \#C-CA=CA \\
\hline 33 & 85 & 120 & \#CA=CA-C1 \\
\hline 34 & 80 & 120.4 & \#CA-C1=O1 \\
\hline 35 & 85 & 120 & \#CA-C1-CA \\
\hline
\end{tabular}


Table A.4: Dihedral Coefficients

\begin{tabular}{|c|c|c|c|c|c|}
\hline $\begin{array}{l}\text { Dihedral } \\
\text { Type }\end{array}$ & $V_{1}$ & $\mathrm{~V}_{2}$ & $V_{3}$ & $\mathrm{~V}_{4}$ & Atom Types \\
\hline 1 & 0 & 0 & 0 & -0.744 & \#CM=CM-CT1-HC1 \\
\hline 2 & 0.692 & 0.81 & -1.808 & 0 & \#CM=CM-CT1-CT1 \\
\hline 3 & 0.692 & 0.81 & -1.808 & 0 & \#CM=CM-CT1-CT0 \\
\hline 4 & 0 & 0 & 0.732 & 0 & \#CM-CT1-CT1-HC1 \\
\hline 5 & 2.6 & -0.1 & 0.4 & 0 & \#CM-CT1-CT1-C \\
\hline 6 & 2.6 & -0.1 & 0.4 & 0 & \#CM-CT1-СТ1-СТ1 \\
\hline 7 & 0 & 0 & 0.732 & 0 & \#CM-CT1-C0-HC1 \\
\hline 8 & 0 & -0.1 & 0.4 & 0 & \#CM-CT1-C0-HC1 \\
\hline 9 & 2.6 & -0.1 & 0.4 & 0 & \#CT1-CT1-CT1-CT1 \\
\hline 10 & 0 & 1.092 & 0 & 0 & \#CT1-CT1-C=O \\
\hline 11 & 6.5 & -0.804 & -0.272 & 0 & \#CT1-CT1-C-N \\
\hline 12 & 0 & 0 & 0.6 & 0 & \#CT1-CT1-CT1-HC1 \\
\hline 13 & -3.394 & -0.912 & 1.17 & 0 & \#СТ1-СТ1-СТ1-С \\
\hline 14 & 0 & 0 & 0.6 & 0 & \#CT1-C0-CT1-HC1 \\
\hline 15 & 2.6 & -0.1 & 0.4 & 0 & \#CT1-C0-СТ1-СТ1 \\
\hline 16 & 6.2 & 12.178 & 0 & 0 & \#CT1-C-N-C \\
\hline 17 & 0 & 0 & 0.6 & 0 & \#CT1-CT1-C0-HC1 \\
\hline 18 & 0 & 28 & 0 & 0 & \#CT1-CM=CM-CT1 \\
\hline 19 & 2.6 & -0.1 & 0.4 & 0 & \#CT1-CT1-CT1-C0 \\
\hline 20 & 0 & 0 & 0.6 & 0 & \#CT1-CT1-CM-HC \\
\hline 21 & 0 & 28 & 0 & 0 & $\mathrm{HHC}-\mathrm{CM}=\mathrm{CM}-\mathrm{HC}$ \\
\hline 22 & 0 & 28 & 0 & 0 & $\# \mathrm{HC}-\mathrm{CM}=\mathrm{CM}-\mathrm{CT} 1$ \\
\hline 23 & 0 & 0 & 0.636 & 0 & \#HC-CM-CT1-HC1 \\
\hline 24 & 0 & 0 & 0.6 & 0 & \#HC-CM-CT1-C0 \\
\hline 25 & 0 & 0 & 0.6 & 0 & \#HC1-CT1-CT1-HC1 \\
\hline 26 & 0 & 0 & 0.6 & 0 & \#HC1-CT1-C0-HC1 \\
\hline
\end{tabular}




\begin{tabular}{|l|l|l|l|l|l|}
\hline 27 & 0 & 0 & -0.152 & 0 & \#HC1-CT1-CT1-C \\
\hline 28 & 0 & 0 & 0 & 0 & \#HC1-CT1-C=O \\
\hline 29 & 0 & 0 & 0 & 0 & \#HC1-CT1-C-N \\
\hline 30 & 0 & 0 & 0.6 & 0 & \#HC1-CT1-CT1-C0 \\
\hline 31 & 0 & 12.178 & 0 & 0 & \#O=C-N-C \\
\hline 32 & 0 & 14.5 & 0 & 0 & \#CA=CA-CA=CA \\
\hline 33 & 0 & 14.5 & 0 & 0 & \#CA=CA-CA-HA \\
\hline 34 & 0 & 0 & 0 & 0 & \#CA=CA-CT0-CA \\
\hline 35 & 0 & 0 & 0 & 0 & \#CA=CA-CT0-HC1 \\
\hline 36 & 0 & 14.5 & 0 & 0 & \#HA-CA=CA-CT0 \\
\hline 37 & 0 & 14.5 & 0 & 0 & \#CA=CA-CA-CT0 \\
\hline 38 & 0 & 14.5 & 0 & 0 & \#HA-CA=CA-HA \\
\hline 39 & 0 & 14.5 & 0 & 0 & \#CA0=CA-CA=CA \\
\hline 40 & 0 & 14.5 & 0 & 0 & \#CA0=CA-CA-HA \\
\hline 41 & 0 & 14.5 & 0 & 0 & \#CA=CA0-CA-CA \\
\hline 42 & 0 & 14.5 & 0 & 0 & \#CA=CA0-CA-HA \\
\hline 43 & 0 & 12.178 & 0 & 0 & \#O=C-N-CA0 \\
\hline 44 & 0 & 4.1 & 0 & 0 & \#C-N-CA0=CA \\
\hline 45 & 4.6 & 12.178 & 0 & 0 & \#CT1-C-N-CA0 \\
\hline 46 & 0 & 14.5 & 0 & 0 & \#N-CA0=CA-CA \\
\hline 47 & 0 & 14.5 & 0 & 0 & \#N-CA0=CA-HA \\
\hline 48 & 1.6 & 0 & 1.8 & 0 & \#C-CT1-CT1-C \\
\hline 49 & -4.12 & -0.626 & 0.63 & 0 & \#C-CT1=CT1-C0 \\
\hline 50 & 4.6 & 12.178 & 0 & 0 & \#CA0-N-C-CA \\
\hline 51 & 0 & 2.2 & 0 & 0 & \#N-C-CA=CA \\
\hline 52 & 0 & 4.2 & 0 & 0 & \#O=C-CA=CA \\
\hline 53 & 0 & 14.5 & 0 & 0 & \#C-CA=CA-C \\
\hline 54 & 0 & 14.5 & 0 & 0 & \#C-CA=CA-CA \\
\hline 55 & 0 & 14.5 & 0 & 0 & \#C-CA=CA-HA \\
\hline
\end{tabular}




\begin{tabular}{|l|l|l|l|l|l|}
\hline 56 & 0 & 14.5 & 0 & 0 & \#HA-CA=CA-C1 \\
\hline 57 & 0 & 4.2 & 0 & 0 & \#CA=CA-C1=O1 \\
\hline 58 & 0 & 14.5 & 0 & 0 & \#CA-CA=CA-C1 \\
\hline 59 & 4.6 & 12.178 & 0 & 0 & \#C-N-C-CA \\
\hline 60 & 0 & 14.5 & 0 & 0 & \#CA-CA-C1-CA \\
\hline
\end{tabular}




\section{Appendix B}

LAMMPS input scripts used for energy minimizations, deform command, create bond command, NVE dynamics and temp rescale

\section{B.1 Molecular Energy Minimization and Deform Simulation \\ \#---------initialization-------------}

units real

dimension 3

boundary $\mathrm{p} \mathrm{p} \mathrm{p}$

atom_style molecular

neighbor $2.0 \mathrm{nsq}$

neigh_modify every 1 delay 0 check yes page 50000000 one 10000

variable density equal mass(all $) /\left(0.602^{*} \mathrm{vol}\right)$

\#-----------force-field---------------

bond_style harmonic

angle_style harmonic

dihedral_style opls

dihedral_coeff 180.013

pair_style lj/cut 10.0

read_data polymer150.txt

\#-------------settings-----------------

timestep 0.5

variable Time equal step ${ }^{*} \mathrm{dt} / 1000$ \#time in picoseconds

variable mass equal mass(all) 
thermo_style custom step $v \_$Time $v \_$density temp press etotal ke pe ebond eangle edihed evdwl v_mass

\#--------------run--------------------

dump 1 all atom 10 molecule_minimization.lammpstrj

dump_modify 1 scale no sort id

thermo 1

fix 1 all viscous 0.999999

fix 2 all temp/rescale 13030301

fix 3 all nve/limit 0.001

compute 4 all pe/atom

fix 5 all deform $1 \mathrm{x}$ final $-32.532 .5 \mathrm{y}$ final $-32.532 .5 \mathrm{z}$ final -32.532 .5 units box run 10000

unfix 1

unfix 2

unfix 3

undump 1

reset_timestep 0

fix 1 all nvt temp 3003001000

fix 2 all momentum 250 linear 111 angular

velocity all create 3004928459 rot yes mom yes

thermo_style custom step v_Time v_density temp pe ke etotal press v_mass

thermo 10

log molecule_dynamics.log.lammps

dump 1 all atom 100 molecule_dynamics.lammpstrj

dump_modify 1 scale no

dump 4 all atom 500 atom_anglenergy.lammpstrj

run 40000

write_restart *molecule1_dynamics.restart 


\section{B.2 Fix Create/Bond Command}

\#----------initialization-------------

units real

dimension 3

boundary $p p p$

atom_style molecular

neighbor 2.0 nsq

neigh_modify every 1 delay 0 check yes page 50000000 one 10000

variable density equal mass $($ all $) /\left(0.602^{*} \mathrm{vol}\right)$

\#-----------force-field--------------

bond_style harmonic

angle_style harmonic

dihedral_style opls

dihedral_coeff 180.013

pair_style lj/cut 10.0

read_data 40000molecule10_dynamics.restart

\#-------------settings----------------

timestep 0.25

variable Time equal step* ${ }^{*} t / 1000$

variable mass equal mass(all)

thermo_style custom step v_Time v_density temp press etotal ke pe ebond eangle edihed evdwl v_mass

\#-------------run-------------------- 
dump 1 all atom 10 molecule11_minimization.lammpstrj

dump_modify 1 scale no sort id

thermo 1

fix 1 all viscous 0.999999

fix 2 all temp/rescale 13030301

fix 3 all nve/limit 0.001

compute 4 all pe/atom

fix 5 all bond/create 514157.019 iparam 12 jparam 12

special_bonds lj 0.01 .01 .0

compute 6 all property/local batom 1 batom 2 btype

compute 7 all bond/local dist eng

variable batom1 equal c_6[1]

variable batom2 equal c_6[2]

variable btype equal c_6[3]

variable dist equal c_7[1]

variable eng equal c_7[2]

run 10000

unfix 1

unfix 2

unfix 3

undump 1

dump 7 all local 10000000 pmr150crossdetail.txt c_6[1] c_6[2] c_6[3] c_7[1]

c_7[2]

reset_timestep 0

fix 1 all nvt temp 3003001000

fix 2 all momentum 250 linear 111 angular

velocity all create 3004928459 rot yes mom yes

thermo_style custom step v_Time v_density temp pe ke etotal press v_mass

thermo 10

log molecule11_dynamics.log.lammps 
dump 1 all atom 100 molecule11_dynamics.lammpstrj

dump modify 1 scale no

dump 4 all atom 500 atom11_anglenergy.lammpstrj

run 40000

write_restart *molecule11_dynamics.restart

\section{B.3 Calculate Density Distribution Data}

\#---------initialization------------

units real

dimension 3

boundary $p p p$

atom_style molecular

neighbor $2.0 \mathrm{nsq}$

neigh_modify every 1 delay 0 check yes page 50000000 one 10000

variable density equal mass(all $) /\left(0.602^{*} \mathrm{vol}\right)$

\#-----------force-field---------------

bond_style harmonic

angle_style harmonic

dihedral_style opls

dihedral_coeff 180.013

pair_style lj/cut 10.0

read_data 40000molecule10_dynamics.restart

\#-------------settings----------------

timestep 0.25 
variable Time equal step* $\mathrm{dt} / 1000$

variable mass equal mass(all)

thermo_style custom step v_Time v_density temp press etotal ke pe ebond eangle edihed evdwl v_mass

\#--------------run--------------------

dump 1 all atom 10 molecule11_minimization.lammpstrj

dump_modify 1 scale no sort id

thermo 1

fix 1 all viscous 0.999999

fix 2 all temp/rescale 13030301

fix 3 all nve/limit 0.001

compute 4 all pe/atom

fix 5 all ave/spatial $10055000 \times-32.50 .005$ density/mass units box ave one file densitydataforX3.txt

fix 6 all ave/spatial 10055000 y -32.50 .005 density/mass units box ave one file densitydataforY3.txt

fix 7 all ave/spatial 10055000 z -32.50 .005 density/mass units box ave one file densitydataforZ3.txt

unfix 1

unfix 2

unfix 3

undump 1

reset_timestep 0

fix 1 all nvt temp 3003001000

fix 2 all momentum 250 linear 111 angular

velocity all create 3004928459 rot yes mom yes

thermo_style custom step v_Time v_density temp pe ke etotal press v_mass 
thermo 10

log molecule11_dynamics.log.lammps

dump 1 all atom 100 molecule11_dynamics.lammpstrj

dump modify 1 scale no

dump 4 all atom 500 atom11_anglenergy.lammpstrj

run 40000

write_restart *molecule11_dynamics.restart

\section{B.4 NVT Simulations}

\#---------initialization-------------

Units real

dimension 3

boundary $p p p$

atom_style molecular

neighbor $2.0 \mathrm{nsq}$

neigh_modify every 1 delay 0 check yes page 50000000 one 10000

variable density equal mass(all)/(0.602*vol)

\#-----------force-field---------------

bond_style harmonic

angle_style harmonic

dihedral_style opls

pair_style lj/cut 10.0

read_restart 40000molecule11_dynamics.restart

\#------------settings----------------

timestep 0.5 
variable Time equal step*dt/1000

variable mass equal mass(all)

thermo_style custom step v_Time v_density temp press etotal ke pe ebond eangle edihed evdwl pxx pyy pzz

thermo_modify flush yes norm yes

\#---------------run---------------------

compute ppa all pe/atom

dump 1 all custom 10000 molecule12_prespat.lammpstrj id type x y z c_ppa dump_modify 1 flush yes

thermo 2000

restart 1000000 *molecule12_dynamics.restart

fix 3 all nvt temp 3003001000

fix 2 all momentum 250 linear 111 angular

run 2000000

run 2000000

run 2000000

run 2000000

run 2000000

run 2000000

run 2000000

run 2000000

run 2000000

run 2000000

\section{B.5 NPT Simulations}

\#----------initialization------------- 
Units real

dimension 3

boundary $\mathrm{p} \mathrm{p} \mathrm{p}$

atom_style molecular

neighbor $2.0 \mathrm{nsq}$

neigh_modify every 1 delay 0 check yes page 50000000 one 10000

variable density equal mass $($ all $) /\left(0.602^{*} \mathrm{vol}\right)$

\#----------force-field---------------

bond_style harmonic

angle_style harmonic

dihedral_style opls

pair_style lj/cut 10.0

read_restart 40000molecule11_dynamics.restart

\#-------------settings----------------

timestep 0.5

variable Time equal step*dt/1000

variable mass equal mass(all)

thermo_style custom step v_Time v_density temp press etotal ke pe ebond eangle edihed evdwl pxx pyy pzz

thermo_modify flush yes norm yes

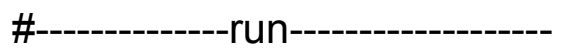

compute ppa all pe/atom

dump 1 all custom 10000 molecule12_prespat.lammpstrj id type x y z c_ppa dump_modify 1 flush yes 
thermo 2000

restart 1000000 *molecule12_dynamics.restart

fix 3 all npt temp 3003001000 iso 1.01 .05000

fix 2 all momentum 250 linear 111 angular

run 2000000

run 2000000

run 2000000

run 2000000

run 2000000

run 2000000 


\section{Appendix C}

Figure 1.3 was recreated with the courtesy of www. wikipedia.org which is provided as author, Zhao WANG's, original work and its copyright released to public. Zhao WANG is a material scientist at the research center of Atomic and Alternative Energies Commission (CEA) in France. The file was uploaded to the website on 2008-03-27 and was released into the Public domain, applicable worldwide. 